\title{
The Maranda Theorem and Liftings of Modules.
}

\author{
S. Ding \\ Ø. Solberg*
}

September 15, 2005

\section{Introduction}

Throughout this paper let $\Lambda$ be a noetherian $R$-algebra where $R$ is a complete local ring with maximal ideal $\underline{m}$ and let $x$ in $\underline{m}$ be a $\Lambda$-regular element. This setting will be referred to as $\Lambda$ being general or the general case. We denote by $\bmod \Lambda$ the category of all finitely generated $\Lambda$-modules.

Let $\Lambda, R$ and $x$ be general and let $M$ be in $\bmod \Lambda$ on which $x$ is regular. Define $n_{x}(M)$ to be the minimal integer $n$ such that $x^{n} \cdot \operatorname{Ext}_{\Lambda}^{1}(M, \quad)=(0)$ if such an integer exists and infinite otherwise. The main object of study in this paper is to prove the following Maranda type of results. Let $M$ be in $\bmod \Lambda$ on which $x$ is regular and assume that $n_{x}(M)$ is finite. If $M / x^{n} M \simeq$ $N / x^{n} N$ for some $N$ in $\bmod \Lambda$ on which $x$ is regular and $n \geq n_{x}(M)+1$, then $M \simeq N$ and if $M$ is indecomposable and $n \geq n_{x}(M)+1$, then $M / x^{n} M$ is indecomposable. In addition we investigate the limit value $n_{x}(M)$ and show that if $M / x^{n} M \simeq N / x^{n} N$ for $M$ indecomposable, $N$ in $\bmod \Lambda$ on which $x$ is regular, $n=n_{x}(M)<\infty$ and $n \geq n_{x}(N)$, then $M \simeq N$ or $N \simeq \Omega_{\Lambda}(M) \simeq$ $\Omega_{\Lambda}^{2}(N)$. Moreover, if $n=n_{x}(M)<\infty$ and $M$ is indecomposable, then $M / x^{n} M$ is indecomposable or $M / x^{n} M \simeq A \oplus \Omega_{\Lambda / x^{n} \Lambda}(A)$, where $A$ is an indecomposable module over $\Lambda / x^{n} \Lambda$. These last two statements are only proved when we in addition assume that $R$ is Gorenstein, such that $\Lambda$ is a maximal CohenMacaulay module over $R$ and $\operatorname{Hom}_{R}\left(\Lambda^{\mathrm{op}}, R\right)$ is isomorphic to $\Lambda$ as left $\Lambda$ modules, and where $M$ in $\bmod \Lambda$ is as an $R$-module a maximal Cohen-Macaulay module over $R$. This setting will be referred to as the Gorenstein case or the

* Supported by the Norwegian Research Council during the preparation of this paper. A.M.S. (1980) subject classification: primary: 16A33, 16A53 secondary: 13H10, 13J10, $13 \mathrm{D} 10$ 
Gorenstein setting. For the more general case we only have weaker results. These Maranda type results and even stronger results are proven for group rings $R G$ in [12], where $R$ is a complete discrete valuation ring of characteristic zero with prime element $p$ and $G$ is a finite group. So this setting is a special case of our Gorenstein case, and in fact [12] is the main source of inspiration for this work. Let $c_{p}$ be the suprenum of $n_{p}(M)$ taken over all $R G$-lattices $M$. This number $c_{p}$ is the same as the highest power $n$ of $p$ such that $p^{n}$ divides the order of $G$ and therefore $c_{p}$ is finite. In [12] the following result besides those also proven in this paper are proved. Let $M$ and $N$ be $R G$-lattices and assume that $M$ is indecomposable and that $M / p^{n} M \simeq N / p^{n} N$ with $n_{p}(M) \geq n_{p}(N)$. If $n=c_{p}$, then $M \simeq N$ unless $p$ divides 2 and the Sylow 2-subgroup of $G$ is of order two [12, Theorem 1.1 (c)]. If $n=c_{p}$, then $M / p^{n} M$ is indecomposable [12, Theorem 1.2 (c)]. For an absolutely indecomposable $R G$-lattice $M$, the number $n_{p}(M)=c_{p}$ if and only if $\operatorname{rank}_{R} M$ is prime to $p$ [12, Theorem 1.4]. We have no analogs of [12, Theorem 1.1 (c) and Theorem 1.4] and [12, Theorem 1.2 (c)] is not true in our more general setting. Further, we have no analog of that the endomorphism ring of $M / p^{n} M$ modulo endomorphisms factoring through projectives is independent of $n$ for $n \geq 2 n_{p}(M)$ [12, Theorem 1.3].

The classical setting in which lattices over orders were studied is the following setting. Let $\Sigma$ be an $S$-order in a finite dimensional separable $K$-algebra $A$, where $S$ is a discrete valuation ring with prime element $\pi$ and quotient ring $K$. In this situation there exists a non-negative integer $i_{0}$ such that $\pi^{i_{0}} \cdot \operatorname{Ext}_{\Sigma}^{1}(M, N)=(0)$ for all $\Sigma$-lattices $M$ and $N$ [4, Cor. 29.5]. This means that the suprenum of $n_{x}(M)$ taken over all $\Sigma$-lattices $M$ is finite. We also study this suprenum of $n_{x}(M)$ for different subcategories and give necessary conditions for it to be finite. In the general case we consider the subcategory $\mathrm{TF}_{x}(\Lambda)$ of all $\Lambda$-modules in $\bmod \Lambda$ on which $x$ is regular. We show that if the suprenum $t_{x}$ of $n_{x}(M)$ taken over $M$ in $\operatorname{TF}_{x}(\Lambda)$ is finite, then $\Lambda_{(x)}$ is semisimple, where $\Lambda_{(x)}$ is the localization of $\Lambda$ with respect to the multiplicative set $\left\{1, x, x^{2}, \ldots\right\}$. For the Gorenstein case we consider the subcategory $\operatorname{CM}_{R}(\Lambda)$ of all $\Lambda$-modules in mod $\Lambda$ which as $R$-modules are Cohen-Macaulay modules over $R$. In this case we prove that if the suprenum $c_{x}$ of $n_{x}(M)$ taken over $M$ in $\mathrm{CM}_{R}(\Lambda)$ is finite, then $\Lambda_{(x)}$ has finite global dimension.

We apply our results to study liftings and weak liftings of modules whose definitions we recall next. Let $\Lambda \rightarrow \Gamma$ be a homomorphism of rings and let $M$ be in $\bmod \Gamma$. Then $L$ in $\bmod \Lambda$ is called a lifting of $M$ if (a) $M \simeq \Gamma \otimes_{\Lambda} L$ and (b) $\operatorname{Tor}_{i}^{\Lambda}(\Gamma, L)=(0)$ for all $i \geq 1$. If $M$ is only a direct summand of $\Gamma \otimes_{\Lambda} L$ for some $\Lambda$-module $L$ satisfying (b), then $L$ is called a weak lifting of $M$ to $\Lambda$. We say that $\mathrm{M}$ is liftable and weakly liftable to $\Lambda$, respectively. We know from [3] that weak liftability is not equivalent to liftability in general, but 
the following result follows easily from our Maranda theorem. Assume that we are in the general case and that the number $t_{x}$ defined above is finite. If $r \geq t_{x}+1$, then a module $C$ in $\bmod \Lambda / x^{r} \Lambda$ is weakly liftable to $\Lambda$ if and only if $C$ is liftable to $\Lambda$. Moreover, every lifting of a $\Lambda$-liftable module in $\bmod \Lambda / x^{r} \Lambda$ is unique up to isomorphism. We are only able to prove a partial converse to this result. Namely, if (in the general case) every lifting of a $\Lambda$-liftable module in $\bmod \Lambda / x^{r} \Lambda$ is unique up to isomorphism, then $r \geq t_{x}$ and in particular $t_{x}$ is finite. Similar results are true in the Gorenstein case.

In all we have done so far elements $x$ in the center of our ring $\Lambda$ which is regular on $\Lambda$ with the property that $x \cdot \operatorname{Ext}_{\Lambda}^{1}(M)=,(0)$ for $\Lambda$-modules $M$ on which $x$ is regular, is of great importance. This section is devoted to studying where we find such elements $x$. In particular we show that if $x$ is in the center of $\Lambda$ and $x \cdot \operatorname{Ext}_{\Lambda}^{1}(M)=,(0)$ for $M$ in $\bmod \Lambda$, then $x$ is in the annihilator of the $\Lambda$-module $I_{M} / I_{M}^{2}$, where $I_{M}$ is the Fitting ideal for $M$ (see section 3 for definition).

Let $A=k\left[\left[z_{0}, \ldots, z_{d}\right]\right]$ be the formal power series ring in the variables $z_{i}$ over an algebraically closed field $k$ of characteristic 0 . The last section is devoted to calculating the numbers $n_{x}()$ and $c_{x}$ for the simple isolated singularities $\mathrm{A}_{n}, \mathrm{D}_{n}, \mathrm{E}_{6}, \mathrm{E}_{7}$ and $\mathrm{E}_{8}$ in $A$ in any dimension.

\section{Reduction modulo a regular element.}

Let $\Lambda$ be an $R$-algebra, where $R$ is a commutative complete local noetherian Gorenstein ring, such that $\Lambda$ is a maximal Cohen-Macaulay module over $R$ and $\operatorname{Hom}_{R}\left(\Lambda^{\mathrm{op}}, R\right)$ is isomorphic to $\Lambda$ as left $\Lambda$-modules. Let $\mathrm{CM}_{R}(\Lambda)$ denote the category of all the finitely generated $\Lambda$-modules which are maximal CohenMacaulay modules over $R$. Let $x$ be a $\Lambda$-regular element in the maximal ideal of $R$. The main aim in this section is to prove the Maranda theorem in the setting above and study what happens for the limit value of the Maranda theorem. Moreover, we show that if the suprenum of $n_{x}(M)$ taken over $M$ in $\mathrm{CM}_{R}(\Lambda)$ is finite, then $\Lambda_{(x)}$ has finite global dimension, where $\Lambda_{(x)}$ is the localization of $\Lambda$ with respect to the multiplicative set $\left\{1, x, x^{2}, \ldots\right\}$. We also consider the general case and we show that if the suprenum of $n_{x}(M)$ taken over $M$ in $\bmod \Lambda$ on which $x$ is regular is finite, then $\Lambda_{(x)}$ is a semisimple ring.

Let $\Lambda$ be as above. For any $M$ in $\operatorname{CM}_{R}(\Lambda)$ we denote by $\Omega_{\Lambda}^{i}(M)$ the $i$-th syzygy of a minimal projective resolution of $M$ over $\Lambda$. In this setting the syzygies of $M$ are indecomposable if and only if $M$ is indecomposable. In order to prove our results we will make use of the following general result. 
Proposition 1.1 ([7]) Let $\Gamma$ be an arbitrary ring and $f: A \rightarrow B$ a homomorphism between two modules in mod $\Gamma$. Then the following are equivalent.

(a) The map $f$ factors through a projective $\Gamma$-module.

(b) $f \cdot \operatorname{Ext}_{\Gamma}^{1}(B)=,(0)$.

(c) $f \cdot\left(0 \rightarrow \Omega_{\Gamma}(B) \rightarrow P \rightarrow B \rightarrow 0\right)=0$ in $\operatorname{Ext}_{\Gamma}^{1}\left(A, \Omega_{\Gamma}(B)\right)$, where $P$ is a projective $\Gamma$-module.

In particular, if $f$ is a monomorphism factoring through a projective and $\Omega_{\Gamma}($ coker $f)$ is the kernel of the map $P \rightarrow B \rightarrow B / \operatorname{Im} f$, then we have that $\Omega_{\Gamma}(\operatorname{coker} f) \simeq A \oplus \Omega_{\Gamma}(B)$.

For the next corollary let $\Lambda, R$ and $x$ be as in the general setting.

Corollary 1.2 Let $x$ in $\underline{m}$ be $\Lambda$-regular and $M$ in $\bmod \Lambda$ such that $x$ is $M$ regular. Then $x \cdot \operatorname{Ext}_{\Lambda}^{1}(M)=,(0)$ if and only if $\Omega_{\Lambda}(M / x M) \simeq M \oplus \Omega_{\Lambda}(M)$.

Proof: Let $P_{M} \rightarrow M$ be the projective cover of $M$. Then $P_{M} \rightarrow M \rightarrow M / x M$ is the projective cover of $M / x M$. Hence we have the following commutative diagram

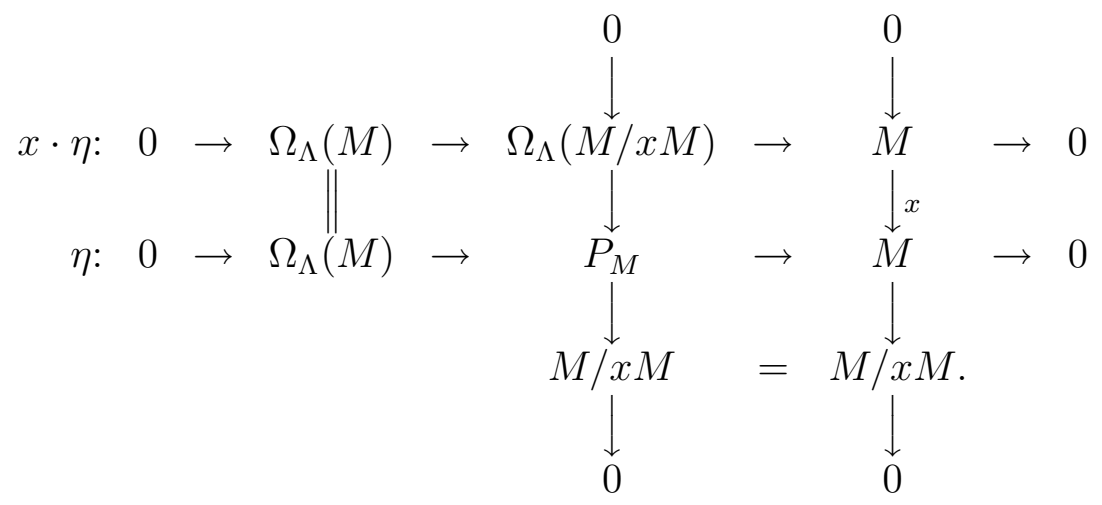

If $x \cdot \operatorname{Ext}_{\Lambda}^{1}(M)=,(0)$, then $\Omega_{\Lambda}(M / x M) \simeq M \oplus \Omega_{\Lambda}(M)$ by Proposition 1.1. If $\Omega_{\Lambda}(M / x M) \simeq M \oplus \Omega_{\Lambda}(M)$, then the sequence $x \cdot \eta$ is split exact by [9] and therefore $x \cdot \operatorname{Ext}_{\Lambda}^{1}(M)=,(0)$ by Proposition 1.1 .

Again, let $\Lambda$ be general. Let $x$ in $\underline{m}$ be a $\Lambda$-regular element and denote by $\mathrm{TF}_{x}(\Lambda)$ the category of all finitely generated $\Lambda$-modules on which $x$ is regular. For $M$ in $\mathrm{TF}_{x}(\Lambda)$ we define

$$
n_{x}(M)=\min \left\{n \mid x^{n} \cdot \operatorname{Ext}_{\Lambda}^{1}(M,)=(0)\right\}
$$

if there exists an $n$ in $\mathbf{N}$ such that $x^{n} \cdot \operatorname{Ext}_{\Lambda}^{1}(M)=,(0)$ and $n_{x}(M)=\infty$ otherwise. Further, for any subcategory $\mathcal{C}$ of $\operatorname{TF}_{x}(\Lambda)$ we define

$$
|\mathcal{C}|_{x}=\sup \left\{n_{x}(M) \mid M \in \mathcal{C}\right\} .
$$

Now we can formulate our main result in this section. 
Theorem 1.3 Let $\Lambda, R$ and $x$ be as in the Gorenstein setting. Assume that $M$ is in $\mathrm{CM}_{R}(\Lambda)$ with $n_{x}(M)$ finite. Then we have the following.

(a) If $M / x^{n} M \simeq N / x^{n} N$ for some $N$ in $\bmod \Lambda$ with $x N$-regular and $n \geq n_{x}(M)+1$, then $M \simeq N$.

Assume in addition that $M$ is indecomposable.

(b) If $M / x^{n} M \simeq N / x^{n} N$ for some $N$ in $\bmod \Lambda$ with $x N$-regular and $n=n_{x}(M) \geq 1$ and $n \geq n_{x}(N)$, then $M \simeq N$ or $N \simeq \Omega_{\Lambda}(M) \simeq \Omega_{\Lambda}^{2}(N)$.

(c) If $n \geq n_{x}(M)+1$, then $M / x^{n} M$ is indecomposable.

(d) If $n=n_{x}(M) \geq 1$, then $M / x^{n} M$ is indecomposable or $M / x^{n} M \simeq$ $A \oplus \Omega_{\Lambda / x^{n} \Lambda}(A)$, where $A$ is an indecomposable module over $\Lambda / x^{n} \Lambda$.

Proof: (a) This proof follows along the lines of the proofs of $[10$, Theorem 1.1] and [3, Theorem 2.9]. Let $M$ be in $\operatorname{CM}_{R}(\Lambda)$ with $n_{x}(M)$ finite. Assume that $\varphi: M / x^{n} M \rightarrow N / x^{n} N$ is an isomorphism for some $N$ in $\operatorname{TF}_{x}(\Lambda)$ and $n \geq n_{x}(M)+1$. Since $n-1 \geq n_{x}(M)$, the map $x^{n-1} \cdot \operatorname{id}_{M}: M \rightarrow M$ factors through a projective $\Lambda$-module $P$. We have the following commutative diagram

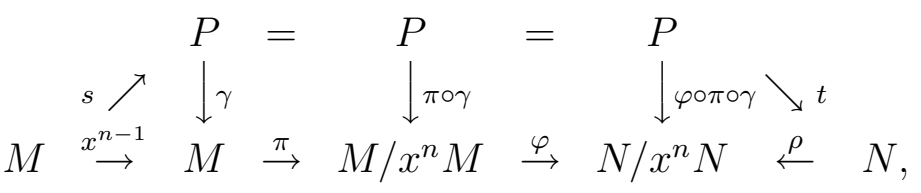

where $\pi: M \rightarrow M / x^{n} M$ and $\rho: N \rightarrow N / x^{n} N$ are the natural epimorphisms. Then there exists a map $t: P \rightarrow N$ such that $\varphi_{\circ} \pi \circ \gamma=\rho \circ$, since $P$ is projective. Hence, the map $\sigma=t_{\circ} s: M \rightarrow N$ has the property that $\rho_{\circ} \sigma=\psi$, where $\psi=$ $\varphi \circ \pi \circ x^{n-1}$. The image of $\psi: M \rightarrow N / x^{n} N$ is $x^{n-1} N / x^{n} N$ and the preimage of $x^{n-1} N / x^{n} N$ under $\rho: N \rightarrow N / x^{n} N$ is $x^{n-1} N$. This implies that $\operatorname{Im} \sigma \subset x^{n-1} N$. Let $C=x^{n-1} N / \operatorname{Im} \sigma$. For any $x^{n-1} a^{\prime}$ in $x^{n-1} N$ there exists an $a$ in $M$ such that $x^{n-1} a^{\prime}=\sigma(a)-x^{n} a^{\prime \prime}$ for some $a^{\prime \prime}$ in $N$. It follows that $C=x C$ and therefore $C=0$ or equivalently $\operatorname{Im} \sigma=x^{n-1} N$ by the Nakayama Lemma. Since $x$ is regular on $N$, we have proved that $\sigma: M \rightarrow x^{n-1} N \simeq N$ is an epimorphism. It remains to show that $\sigma$ is a monomorphism. Assume that $\sigma(m)=0$ for some $m$ in $M$. Then $0=\psi(m)=\rho \circ \sigma(m)=\varphi\left(\pi\left(x^{n-1} m\right)\right)$. By assumption the map $\varphi$ is an isomorphism, so that $x^{n-1} m=x^{n} m_{1}$ for some $m_{1}$ in $M$. Since $x$ is regular on both $M$ and $N$, we have $m=x m_{1}$ and $\sigma\left(m_{1}\right)=0$. So by continuing this process the element $m$ is in $\cap_{i=1}^{\infty} x^{i} M=(0)$. Hence $\sigma$ is a monomorphism and $M$ and $N$ are isomorphic.

(b) Assume that $M$ in $\mathrm{CM}_{R}(\Lambda)$ is indecomposable with $n_{x}(M)$ finite and $n_{x}(M) \geq 1$. Suppose that $M / x^{n} M \simeq N / x^{n} N$ for some $N$ in $\operatorname{TF}_{x}(\Lambda)$ with $n=n_{x}(M)$ and $n \geq n_{x}(N)$. Then by Corollary 1.2 we have that

$$
\begin{aligned}
\Omega_{\Lambda}\left(M / x^{n} M\right) & \simeq M \oplus \Omega_{\Lambda}(M) \\
\| \chi & \\
\Omega_{\Lambda}\left(N / x^{n} N\right) & \simeq N \oplus \Omega_{\Lambda}(N) .
\end{aligned}
$$


Since $M$ and $\Omega_{\Lambda}(M)$ are indecomposable, it follows that $M \simeq N$ or $N \simeq$ $\Omega_{\Lambda}(M) \simeq \Omega_{\Lambda}^{2}(N)$.

(c) Assume that $M$ in $\mathrm{CM}_{R}(\Lambda)$ is indecomposable with $n_{x}(M)$ finite and that $n \geq n_{x}(M)+1$. We want to prove that $M / x^{n} M$ is indecomposable. Suppose $M / x^{n} M \simeq C \oplus C^{\prime}$ is a non-trivial decomposition and let $\varphi: M / x^{n} M \rightarrow$ $M / x^{n} M$ be the idempotent corresponding to $C$ in this decomposition. Since $n-1 \geq n_{x}(M)$, we can use the same arguments as in (a) to construct the following commutative diagram

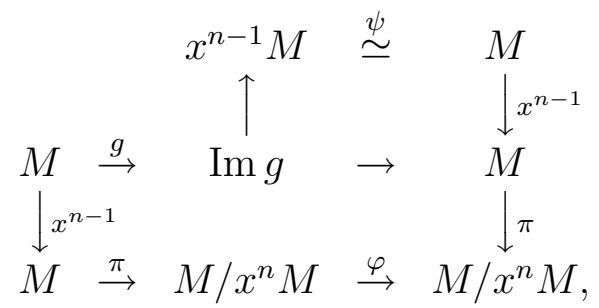

where $g: M \rightarrow M$ corresponds to the map tos in (a). Let $\psi: x^{n-1} M \rightarrow M$ be the inverse of the isomorphism $x^{n-1}: M \rightarrow x^{n-1} M$ and let $h=\psi \circ g$. Then using that $M / x M \stackrel{x^{n-1}}{\rightarrow} x^{n-1} M / x^{n} M$ is an isomorphism, it is easy to see that we have the following commutative diagram

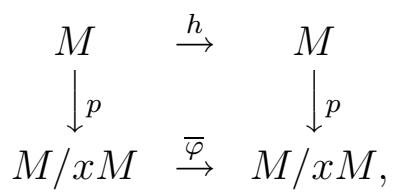

where $p: M \rightarrow M / x M$ is the natural epimorphism and $\bar{\varphi}: M / x M \rightarrow M / x M$ denotes the homomorphism induced by $\varphi$. If $\bar{\varphi}=0$, then $\operatorname{Im} \varphi \subset x M$ and therefore $\operatorname{Im} \varphi=\operatorname{Im} \varphi^{2} \subset x^{2} M$. This implies that $\varphi=0$, which is a contradiction since the decomposition is non-trivial. If $\bar{\varphi}=\mathrm{id}_{M / x M}$, we apply the previous argument to $\operatorname{id}_{M / x M}-\bar{\varphi}$ and deduce the same contradiction. Hence $\bar{\varphi}$ is a non-trivial idempotent. Since $\bar{\varphi}$ is an idempotent, $h-h^{2}=x h^{\prime}$ for some $h^{\prime}$ in $\Gamma=\operatorname{End}_{\Lambda}(M)$. Therefore $h$ is an idempotent in $\Gamma / \underline{m} \Gamma$. Since $M$ is a finitely generated $\Lambda$-module and $\Lambda$ is a noetherian $R$-algebra, $\Gamma$ is also a noetherian $R$-algebra and therefore complete in the $\underline{m} \Gamma$-adic topology. Then by $[4$, Theorem 6.7 (i) $] h$ in $\Gamma / \underline{m} \Gamma$ can be lifted to an idempotent $\tilde{h}$ in $\Gamma$. The idempotent $\tilde{h}$ can not be trivial, since $\bar{\varphi}$ is non-trivial and therefore $M$ is decomposable. This is contradicting our assumption on $M$, therefore $M / x^{n} M$ is indecomposable for $n \geq n_{x}(M)+1$.

(d) Assume that $M$ in $\mathrm{CM}_{R}(\Lambda)$ is indecomposable and that $n=n_{x}(M) \geq 1$ is finite. Suppose $M / x^{n} M$ decomposes, say $M / x^{n} M \simeq B \oplus B^{\prime}$. Since $n=$ $n_{x}(M)$, we have that $\Omega_{\Lambda}\left(M / x^{n} M\right) \simeq M \oplus \Omega_{\Lambda}(M)$ by Corollary 1.2. Since 
$\Omega_{\Lambda}\left(M / x^{n} M\right) \simeq \Omega_{\Lambda}(B) \oplus \Omega_{\Lambda}\left(B^{\prime}\right)$ and $M$ and $\Omega_{\Lambda}(M)$ are indecomposable, we have that $M \simeq \Omega_{\Lambda}(B)$ after maybe interchanging $B$ and $B^{\prime}$. Since $B$ is weakly liftable, we have that $M / x^{n} M \simeq \Omega_{\Lambda}(B) / x^{n} \Omega_{\Lambda}(B) \simeq B \oplus \Omega_{\Lambda / x^{n} \Lambda}(B)$ by $[3$, Prop. 3.2]. Since $M \simeq \Omega_{\Lambda}(B)$ is indecomposable, $B$ is indecomposable.

Remark: Let $\Lambda$ be general. Theorem 1.3 (a) and (c) are still true in this setting if $M$ and $N$ are in $\operatorname{TF}_{x}(\Lambda)$. In the rest of this remark let $M$ in $\mathrm{TF}_{x}(\Lambda)$ be indecomposable and assume that $1 \leq n_{x}(M)<\infty$. In this setting Theorem 1.3 (b) must be replaced with the following: If $M / x^{n} M \simeq N / x^{n} N$ for some $N$ in $\bmod \Lambda$ with $x N$-regular and $n=n_{x}(M)$ and $n \geq n_{x}(N)$, then $M \simeq N$ or $\Omega_{\Lambda}(M) \simeq N \oplus X$ and $\Omega_{\Lambda}(N) \simeq M \oplus X$. Theorem 1.3 (d) must be replaced in this setting by: If $n=n_{x}(M)$, then $M / x^{n} M$ is indecomposable or $M / x^{n} M \simeq B \oplus B^{\prime}$, where $B$ is an indecomposable module over $\Lambda / x^{n} \Lambda$ and $B^{\prime}$ is a direct summand of $\Omega_{\Lambda / x^{n} \Lambda}(B)$. In the setting of the theorem the similar statement as in [12, Theorem 1.2 (c)] namely, if $c_{x}=\left|\mathrm{CM}_{R}(\Lambda)\right|_{x}<\infty$ and $M$ in $\mathrm{CM}_{R}(\Lambda)$ is indecomposable, then $M / x^{n} M$ is indecomposable, is not true in general. One counterexample is the $\mathrm{A}_{2}$-singularity in dimension 1 , which we will give in section 2 .

The proof of this result for group rings $R G$ over a complete discrete valuation ring $R$ in [12], is based on the following. Let $p$ be the generator of the maximal ideal of $R$. If $M$ is an $R G$-lattice and $n \geq n_{p}(M)$, then the minimal Cohen-Macaulay approximation (See [2] and [1]) of $M / p^{n} M$ is isomorphic to $M \oplus \Omega_{\Lambda}^{-1}(M)$ modulo projectives. In addition some knowledge about the endomorphism ring of $M / p^{n} M$ was used. The result concerning the minimal Cohen-Macaulay approximation is true in our setting. Let $\Lambda, R$ and $x$ be as in the Gorenstein setting. Then for any $M$ in $\bmod \Lambda$ there exists an exact sequence

$$
0 \rightarrow Y_{M} \rightarrow X_{M} \stackrel{\alpha}{\rightarrow} M \rightarrow 0
$$

where $X_{M}$ is in $\operatorname{CM}_{R}(\Lambda), Y_{M}$ has finite projective dimension and whenever $\alpha=\alpha \circ g$ for $g: X_{M} \rightarrow X_{M}$ the map $g$ is an automorphism [1]. The module $X_{M}$ is called the minimal Cohen-Macaulay approximation of $M$. We also need the following additional facts. A $\Lambda$-module $M$ is in $\operatorname{CM}_{R}(\Lambda)$ if and only if $\operatorname{Ext}_{\Lambda}^{i}(M, \Lambda)=0$ for all $i>0$. Moreover, for any module $M$ in $\mathrm{CM}_{R}(\Lambda)$ there exists an exact sequence $0 \rightarrow M \stackrel{f}{\rightarrow} P \rightarrow M^{\prime} \rightarrow 0$ with $P$ a projective $\Lambda$ module and $M^{\prime}$ in $\operatorname{CM}_{R}(\Lambda)$. If the map $f$ is minimal, then we denote $M^{\prime}$ by $\Omega_{\Lambda}^{-1}(M)$. If $0 \rightarrow M \rightarrow Q \rightarrow N \rightarrow 0$ is exact with $M$ and $N$ in $\operatorname{CM}_{R}(\Lambda)$ and $Q$ a projective $\Lambda$-module, then $N \simeq \Omega_{\Lambda}^{-1}(M) \oplus Q^{\prime}$ for some projective $\Lambda$-module $Q^{\prime}$. With these preliminaries we have the following. 
Proposition 1.4 Let $\Lambda$ and $R$ be as in the Gorenstein setting with $x$ in $\underline{m}$ regular on $\Lambda$. Let $M$ be in $\mathrm{CM}_{R}(\Lambda)$ with no non-zero projective summands. Then $x \cdot \operatorname{Ext}_{\Lambda}^{1}(M)=$,0 if and only if $X_{M / x M} \simeq M \oplus \Omega_{\Lambda}^{-1}(M)$, where $X_{M / x M}$ is the minimal Cohen-Macaulay approximation of $M / x M$.

Proof: Let $0 \rightarrow Y_{M / x M} \rightarrow X_{M / x M} \rightarrow M / x M \rightarrow 0$ be the minimal CohenMacaulay approximation of $M / x M$. Then $Y_{M / x M}$ is a projective $\Lambda$-module (see [3, Remarks after Prop. 4.4]). From [3, Proposition 4.1] we have the following commutative diagram

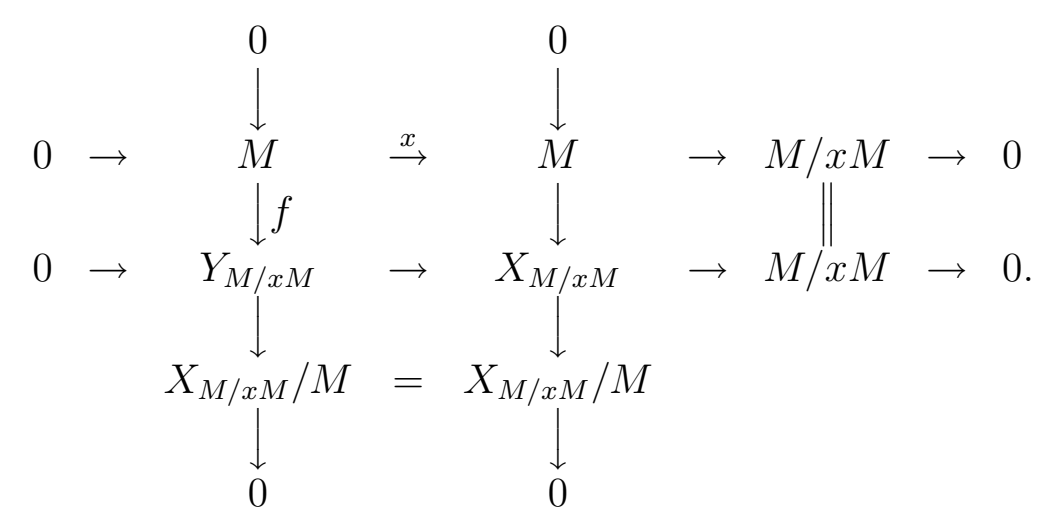

Applying the functor $\Lambda / x \Lambda \otimes_{\Lambda}$ - to the above diagram shows that the reduction of the middle vertical sequence is split, since $x$ is regular on $X_{M / x M} / M$ by [3, Proposition 4.2]. Hence

$$
X_{M / x M} / x X_{M / x M} \simeq M / x M \oplus\left(X_{M / x M} / M\right) / x\left(X_{M / x M} / M\right)
$$

and therefore we have that $X_{M / x M} / M$ is in $\mathrm{CM}_{R}(\Lambda)$. Since $Y_{M / x M}$ is projective and $X_{M / x M} / M$ is in $\mathrm{CM}_{R}(\Lambda)$, it follows that $X_{M / x M} / M \simeq \Omega_{\Lambda}^{-1}(M) \oplus P$ for some projective $\Lambda$-module $P$.

Assume that $x \cdot \operatorname{Ext}_{\Lambda}^{1}(M)=,(0)$. We want to show that the middle vertical sequence in the diagram above splits and that $P=(0)$. We have that $x \cdot \operatorname{Ext}_{\Lambda}^{1}(M)=,(0)$ is equivalent to that $x \cdot \mathrm{id}_{M}: M \rightarrow M$ factors through a projective by Proposition 1.1. Since $\operatorname{Ext}_{\Lambda}^{1}\left(X_{M / x M} / M, \Lambda\right)=0$, the map $x \cdot \mathrm{id}_{M}$ factors through the map $f: M \rightarrow Y_{M / x M}$. This implies that the pushout of the leftmost vertical sequence in the above diagram is split. Therefore the middle vertical sequence in the diagram above splits and $X_{M / x M} \simeq M \oplus X_{M / x M} / M \simeq M \oplus \Omega_{\Lambda}^{-1}(M) \oplus P$. Since $M / x M$ is weakly liftable to $\Lambda$, we have that $X_{M / x M} / x X_{M / x M} \simeq M / x M \oplus \Omega_{\Lambda / x \Lambda}^{-1}(M / x M)$ by [3, Proposition 4.5]. Because $\Omega_{\Lambda}^{-1}(M) / x \Omega_{\Lambda}^{-1}(M) \simeq \Omega_{\Lambda / x \Lambda}^{-1}(M / x M)$, the module $P$ is zero and $X_{M / x M} \simeq M \oplus \Omega_{\Lambda}^{-1}(M)$.

Assume that $X_{M / x M} \simeq M \oplus \Omega_{\Lambda}^{-1}(M)$. From the above we have that $X_{M / x M} / M \simeq \Omega_{\Lambda}^{-1}(M) \oplus P$. Therefore $P$ must be a direct summand of 
$X_{M / x M} \simeq M \oplus \Omega_{\Lambda}^{-1}(M)$. Since $M$ has no projective summands, $P$ is a direct summand of $\Omega_{\Lambda}^{-1}(M)$, which is impossible unless $P=(0)$. By [9] the middle vertical sequence in the diagram above splits and therefore $x \cdot \operatorname{id}_{M}: M \rightarrow M$ factors through $f: M \rightarrow Y_{M / x M}$. Hence $x \cdot \operatorname{Ext}_{\Lambda}^{1}(M)=,(0)$ by Proposition 1.1.

Remark: Now one can prove Theorem 1.3 by using this result just by substituting $X_{M / x^{n} M}$ for $\Omega_{\Lambda}\left(M / x^{n} M\right)$ and $\Omega^{-i}$ for $\Omega^{i}$.

Let $\Lambda, R$ and $x$ be as in Proposition 1.4 and assume that $c_{x}=\left|\mathrm{CM}_{R}(\Lambda)\right|_{x}$ is finite. Using Proposition 1.4 we can give a characterization of when $M / x^{c_{x}} M$ is decomposable for $M$ in $\mathrm{CM}_{R}(\Lambda)$ with $n_{x}(M)=c_{x}$ in terms of the minimal Cohen-Macaulay approximation of $M / x^{c_{x}} M$.

Corollary 1.5 Let $\Lambda, R$ and $x$ be as above and assume that $c_{x}=\left|\mathrm{CM}_{R}(\Lambda)\right|_{x}$ is finite. Assume that $M$ in $\mathrm{CM}_{R}(\Lambda)$ is an indecomposable nonprojective module and that $n_{x}(M)=c_{x}$. Then $M / x^{c_{x}} M$ is decomposable if and only if $M$ is the minimal Cohen-Macaulay approximation of a weakly liftable Cohen-Macaulay $\Lambda / x^{c_{x}} \Lambda$-module over $\Lambda$.

Proof: Suppose $M / x^{c_{x}} M$ is decomposable, then $M / x^{c_{x}} M \simeq A \oplus B$ by Theorem $1.3(\mathrm{~d})$, where $A$ and $B$ are indecomposable modules in $\mathrm{CM}_{R / x^{c_{x}}}\left(\Lambda / x^{c_{x}} \Lambda\right)$. From Proposition 1.4 we have that the minimal Cohen-Macaulay approximation of $M / x^{c_{x}} M$ is $M \oplus \Omega_{\Lambda}^{-1}(M)$. Therefore, after maybe interchanging $A$ and $B$, the module $M$ is the minimal Cohen-Macaulay approximation of $A$ over $\Lambda$. Clearly, $A$ is weakly liftable to $\Lambda$.

On the other hand, suppose $M$ is the minimal Cohen-Macaulay approximation of $A$ over $\Lambda$, where $A$ is in $\mathrm{CM}_{R / x^{c_{x}} R}\left(\Lambda / x^{c_{x}} \Lambda\right)$ and weakly liftable to $\Lambda$. Then from [3, Proposition 4.5] we have that $M / x^{c_{x}} M \simeq A \oplus \Omega_{\Lambda / x^{c_{x}} \Lambda}^{-1}(A)$. Therefore $M / x^{c_{x}} M$ is decomposable since $A$ is not a projective $\Lambda / x^{c_{x}} \Lambda$-module.

Remark: Let $\Lambda=R G$, where $R$ is a complete discrete valuation ring of characteristic zero with prime element $p$ and $G$ is a finite group. Then Corollary 1.5 shows that if $M / p^{c_{p}} M$ is decomposable for an indecomposable $R G$-lattice $M$, then $\operatorname{rank}_{R} M=\operatorname{rank}_{R} P$ for some projective $R G$-lattice $P$. Since $|G|$ divides $\operatorname{rank}_{R} P$ for any projective $R G$-lattice $P$ [4, Theorem 29.18], we have that $p^{c_{p}}$ divides $\operatorname{rank}_{R} M$. In [12] this restriction on the rank of $M$ over $R$ was used to show that $M / p^{c_{p}} M$ is indecomposable for any indecomposable $R G$-lattice $M$.

We end this section by characterizing when the number $n_{x}(M)$ is finite and by giving necessary conditions for the numbers $\left|\mathrm{TF}_{x}(\Lambda)\right|_{x}$ and $\left|\mathrm{CM}_{R}(\Lambda)\right|_{x}$ to be finite. Moreover, we give some properties of the function $n_{x}(): \bmod \Lambda \rightarrow \mathbf{N}$. 
First let $\Lambda, R$ and $x$ be as in the general setting. Denote by $M_{(x)}$ the localization of $M$ in $\bmod \Lambda$ with respect to the multiplicative set $\left\{1, x, x^{2}, \ldots\right\}$.

Proposition 1.6 Let $\Lambda, R$ and $x$ be as above.

(a) Let $M$ be in $\mathrm{TF}_{x}(\Lambda)$. Then $n_{x}(M)$ is finite if and only if $M_{(x)}$ is a projective $\Lambda_{(x)}$-module.

(b) If the number $\left|\mathrm{TF}_{x}(\Lambda)\right|_{x}$ is finite, then $\Lambda_{(x)}$ is semisimple.

Proof: (a) Let $M$ and $N$ be in $\bmod \Lambda$. Since $\left(\operatorname{Ext}_{\Lambda}^{1}(M, N)\right)_{(x)} \simeq \operatorname{Ext}_{\Lambda_{(x)}}^{1}\left(M_{(x)}, N_{(x)}\right)$ and $A_{(x)}=(0)$ for a $\Lambda$-module $A$ if and only if $x^{n} A=(0)$ for some finite $n$ in $\mathbf{N}$, we have that $n_{x}(M)$ is finite if and only if $\operatorname{Ext}_{\Lambda_{(x)}}^{1}\left(M_{(x)}, N_{(x)}\right)=(0)$ for all $N$ in $\bmod \Lambda$. Let $B$ be arbitrary in $\bmod \Lambda_{(x)}$ and let $\left\{b_{i}\right\}_{i=1}^{t}$ be a set of generators for $B$ over $\Lambda_{(x)}$. Let $B^{\prime}$ be the $\Lambda$-module $\sum_{i=1}^{t} \Lambda \cdot b_{i}$, then $B_{(x)}^{\prime}=B$. Hence, it follows that $n_{x}(M)$ is finite if and only if $M_{(x)}$ is a projective $\Lambda_{(x)}$-module.

(b) Let $N$ be arbitrary in $\bmod \Lambda_{(x)}$ and let $\left\{n_{i}\right\}_{i=1}^{t}$ be a set of generators for $N$ over $\Lambda_{(x)}$. Let $N^{\prime}$ be the $\Lambda$-module $\sum_{i=1}^{t} \Lambda \cdot n_{i}$. Since $N^{\prime}$ is a subset of $N$ and $x$ is invertible in $\Lambda_{(x)}$, it follows that $x$ is regular on $N^{\prime}$. Hence, $N^{\prime}$ is in $\mathrm{TF}_{x}(\Lambda)$ and $N_{(x)}^{\prime}=N$. Because $n_{x}\left(M^{\prime}\right) \leq\left|\mathrm{TF}_{x}(\Lambda)\right|_{x}<\infty$, the module $N=N_{(x)}^{\prime}$ is projective by (a). This shows that all finitely generated $\Lambda_{(x)^{-}}$ modules are projective. Let $I$ be a submodule of $\Lambda_{(x)}$. Since $\Lambda_{(x)} / I$ is a finitely generated $\Lambda_{(x)}$-module, it follows that $I$ is a direct summand of $\Lambda_{(x)}$. This shows that $\Lambda_{(x)}$ is a semisimple ring.

Remark: Let $\Sigma$ be an $S$-order in a finite dimensional separable $K$-algebra $A$, where $S$ is a discrete valuation ring with prime element $\pi$ and quotient ring $K$. Then there exists a non-negative integer $i_{0}$ such that $\pi^{i_{0}} \cdot \operatorname{Ext}_{\Sigma}^{1}(M, N)=(0)$ for all $\Sigma$-lattices $M$ and $N$ [4, Cor. 29.5]. Since a $\Sigma$-lattice is the same as a $\Sigma$-module on which $\pi$ is regular, it follows that $\left|\operatorname{TF}_{\pi}(\Sigma)\right|_{\pi}$ is finite. A special case of this is a group ring $S G$ where $S$ is a complete discrete valuation ring with maximal ideal generated by a prime number $p$, the group $G$ is finite, the characteristic of $S$ is zero and the characteristic of the residue class field $S /(p)$ is $p$. This was the setting in [12] and here $\left|\mathrm{TF}_{p}(S G)\right|_{p}=n$, where $p^{n}$ is the highest power of $p$ dividing $|G|$. In fact we have the following partial converse. Let $\Lambda, R$ and $x$ be as in the proposition above, but in addition assume that $R$ is contained in $\Lambda$. Assume that $\left|\mathrm{TF}_{x}(\Lambda)\right|_{x}$ is finite. Then $\Lambda_{(x)}$ is semisimple and therefore a product of full matrix rings of division rings. The center $Z\left(\Lambda_{(x)}\right)$ of $\Lambda_{(x)}$ is a product of fields. Since $x$ is $\Lambda$-regular, $R_{(x)}$ is contained in $Z\left(\Lambda_{(x)}\right)$. Therefore $R_{(x)}$ is an artinian ring and $R$ must be of dimension 1. So if $\left|\mathrm{TF}_{x}(\Lambda)\right|_{x}$ is finite, then $\Lambda$ must be a noetherian $R$-algebra, where $R$ is a commutative complete local ring of dimension 1 .

Now, we give some elementary properties of the function $n_{x}(): \bmod \Lambda \rightarrow \mathbf{N}$. 
Proposition 1.7 (a) Let $0 \rightarrow A \rightarrow B \rightarrow C \rightarrow 0$ be an exact sequence in $\bmod \Lambda$. Assume that either $n_{x}(A)$ and $n_{x}(C)$ are finite or $n_{x}(B)$ and $n_{x}(C)$ are finite, then $n_{x}(B) \leq n_{x}(A)+n_{x}(C)$ or $n_{x}(A)$ is finite, respectively.

(b) Let $A$ be in $\bmod \Lambda$ and assume that $n_{x}(A)$ is finite. Then $n_{x}\left(\Omega_{\Lambda}(A)\right) \leq$ $n_{x}(A)$.

(c) Let $A \simeq \oplus_{i=1}^{s} A_{i}$. Then $n_{x}(A)=\max \left\{n_{x}\left(A_{i}\right)\right\}$.

Proof: (a) The last part of (a) follows directly using Proposition 1.6 (a). The first part follows using that we have the exact sequence

$$
\cdots \rightarrow \operatorname{Ext}_{\Lambda}^{1}(C,) \rightarrow \operatorname{Ext}_{\Lambda}^{1}(B,) \rightarrow \operatorname{Ext}_{\Lambda}^{1}(A,) \rightarrow \cdots .
$$

(b) Assume that $x^{n} \cdot \operatorname{id}_{A}: A \rightarrow A$ factors through a projective. In particular it factors through the projective cover of $A$. Hence we have the following diagram

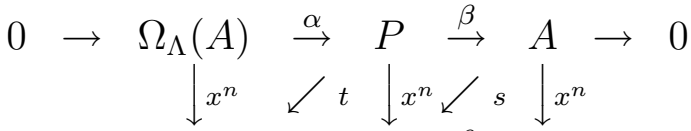

$$
\begin{aligned}
& 0 \rightarrow \Omega_{\Lambda}(A) \stackrel{\alpha}{\rightarrow} P \stackrel{\beta}{\rightarrow} A \rightarrow 0,
\end{aligned}
$$

where $x^{n} \cdot \operatorname{id}_{A}=\beta_{\circ} s$. Then it follows that there exists a map $t: P \rightarrow \Omega_{\Lambda}(A)$, such that $x^{n} \cdot \operatorname{id}_{P}=s \circ \beta+\alpha \circ$. Further this implies that $x^{n} \cdot \operatorname{id}_{\Omega_{\Lambda}(A)}=t_{\circ} \alpha$, hence $n_{x}\left(\Omega_{\Lambda}(A)\right) \leq n$.

(c) This follows directly from the fact that $\operatorname{Ext}_{\Lambda}^{1}(A,) \simeq \oplus_{i=1}^{s} \operatorname{Ext}_{\Lambda}^{1}\left(A_{i}, \quad\right)$.

Let $\Lambda$ and $R$ be as in the Gorenstein setting. If $M$ is in $\operatorname{CM}_{R}(\Lambda)$, then $\operatorname{Ext}_{\Lambda}^{1}(M, \Lambda)=0$. Using this fact we have the following stronger version of Proposition 1.6 (b).

Corollary 1.8 Let $\Lambda, R$ and $x$ be as in the Gorenstein case. If $M$ is in $\mathrm{CM}_{R}(\Lambda)$, then $n_{x}(M)=n_{x}\left(\Omega_{\Lambda}(M)\right)$.

Now, let $\Lambda, R$ and $x$ be as in the Gorenstein setting. Then $R_{(x)}$ is a Gorenstein ring, but not necessarily local, so $R_{(x)}$ Gorenstein means that every localization $\left(R_{(x)}\right)_{\underline{a}}$ is Gorenstein for every maximal ideal $\underline{a}$ in $R_{(x)}$. But still $R_{(x)}$ is Gorenstein if and only if $\mathrm{id}_{R_{(x)}} R_{(x)}<\infty$. A maximal Cohen-Macaulay module $M$ over $R_{(x)}$ is an $R_{(x)}$-module such that $\left(M_{(x)}\right)_{\underline{a}}$ is a maximal Cohen-Macaulay module over $\left(R_{(x)}\right)_{a}$ for every maximal ideal $\underline{a}$ in $R_{(x)}$. This is equivalent to that $\operatorname{Ext}_{R_{(x)}}^{i}\left(M, R_{(x)}\right)=(0)$ for all $i \geq 1$. Let $\mathrm{CM}_{R_{(x)}}\left(\Lambda_{(x)}\right)$ denote the category of finitely generated $\Lambda_{(x)}$-modules which are maximal Cohen-Macaulay modules over $R_{(x)}$. Since we are assuming that $\Lambda$ is a maximal Cohen-Macaulay module over $R$, it follows that $\Lambda_{(x)}$ is in $\operatorname{CM}_{R_{(x)}}\left(\Lambda_{(x)}\right)$ and therefore also every projective $\Lambda_{(x)}$-module. Then it follows that syzygies over $\Lambda_{(x)}$ become 
maximal Cohen-Macaulay modules over $R_{(x)}$, since $\operatorname{id}_{R_{(x)}} R_{(x)}<\infty$. We have that $\operatorname{Hom}_{R_{(x)}}\left(, R_{(x)}\right)$ is a duality on the category of maximal Cohen-Macaulay modules over $R_{(x)}$. The functor $\operatorname{Hom}_{R_{(x)}}\left(, R_{(x)}\right): \mathrm{CM}_{R_{(x)}}\left(\Lambda_{(x)}^{\mathrm{op}}\right) \rightarrow \mathrm{CM}_{R_{(x)}}\left(\Lambda_{(x)}\right)$ extends to a duality. Since we are assuming that ${ }_{\Lambda} \operatorname{Hom}_{R}\left(\Lambda^{\mathrm{op}}, R\right) \simeq{ }_{\Lambda} \Lambda$ and therefore also have $\Lambda_{(x)} \operatorname{Hom}_{R_{(x)}}\left(\Lambda_{(x)}^{\mathrm{op}}, R_{(x)}\right) \simeq \Lambda_{(x)} \Lambda_{(x)}$, it follows that every module in $\mathrm{CM}_{R_{(x)}}\left(\Lambda_{(x)}\right)$ can be considered as a $n$-th syzygy of some module for any $n$. With these preliminaries we show the following.

Proposition 1.9 Let $\Lambda, R$ and $x$ be as in the Gorenstein case. If $\left|\mathrm{CM}_{R}(\Lambda)\right|_{x}$ is finite, then $\Lambda_{(x)}$ has finite global dimension.

Proof: Let $N$ be in $\mathrm{CM}_{R_{(x)}}\left(\Lambda_{(x)}\right)$ be arbitrary. Then $N$ can be considered as $n$-th syzygy of some module in $\mathrm{CM}_{R_{(x)}}\left(\Lambda_{(x)}\right)$ for any $n$, in particular for $n=\operatorname{dim} R_{(x)}$. Assume that $N \simeq \Omega_{\Lambda_{(x)}}^{n}(A)$ for some $A$ in $\mathrm{CM}_{R_{(x)}}\left(\Lambda_{(x)}\right)$. Let $\left\{a_{i}\right\}_{i=1}^{t}$ be a set of generators for $A$ over $\Lambda_{(x)}$ and let $A^{\prime}=\sum_{i=1}^{t} \Lambda \cdot a_{i}$ and $M=\Omega_{\Lambda}^{n}\left(A^{\prime}\right)$. Since $A_{(x)}^{\prime}=A$ and localization is an exact functor preserving projective modules, it follows that $N$ is a direct summand of $M_{(x)}$, where $M$ is in $\mathrm{CM}_{R}(\Lambda)$. Therefore we have shown that if $\left|\mathrm{CM}_{R}(\Lambda)\right|_{x}$ is finite, then every module in $\mathrm{CM}_{R_{(x)}}\left(\Lambda_{(x)}\right)$ is a projective $\Lambda_{(x)}$-module. Since the syzygies $\Omega_{\Lambda_{(x)}}^{i}(B)$ for any module $B$ in $\bmod \Lambda_{(x)}$ become Cohen-Macaulay modules over $R_{(x)}$ for $i \geq \operatorname{dim} R_{(x)}$, it follows that if $\left|\mathrm{CM}_{R}(\Lambda)\right|_{x}$ is finite, then $\Lambda_{(x)}$ has finite global dimension.

Let $S$ be a local Cohen-Macaulay analytic algebra over a perfect field $k$ (that is, it is a homomorphic image of a convergent power series ring over $k$ ). We know that there exists an ideal $\underline{a}$ of $S$ which defines the singular locus of $S$, which means that for a prime ideal $p$ of $S$, the localization $S_{p}$ is regular if and only if $p$ does not contain $\underline{a}[13,6.12]$. Moreover, this ideal $\underline{a}$ has the property that $\underline{a} \cdot \operatorname{Ext}_{S}^{1}(M)=,(0)$ for all $M$ in $\operatorname{CM}(S)[13,6.8]$. Also, an ideal defining the singular locus for an isolated singularity is $\underline{m}$-primary. Combining these results and Proposition 1.9, we have the following.

Corollary 1.10 Let $S$ be as above. The following statements are equivalent.

(a) $S$ is an isolated singularity.

(b) $|\mathrm{CM}(S)|_{x}$ is finite for any regular element $x$ in the maximal ideal of $S$.

(c) There exists a system of parameters $\left\{x_{1}, \ldots, x_{d}\right\}$ of $S$ such that $|\mathrm{CM}(S)|_{x_{i}}$ is finite for $i=1, \ldots, d$.

Example: Let $S$ be a 2-dimensional integrally closed local domain. Suppose its completion with respect to the maximal ideal of $S$ is a homomorphic image of a formal power series ring over a perfect field. Then localizing at any 
element $x$ in the maximal ideal of $S$ we get a ring of finite global dimension, since $S_{(x)}$ is a 1-dimensional integrally closed domain, hence a Dedekind ring. Therefore $|\mathrm{CM}(S)|_{x}$ is finite for every element of the maximal ideal of $S$.

\section{Applications.}

We know in general that weak liftability is not equivalent to liftability in the situation $\Lambda \rightarrow \Lambda / x \Lambda$ for a ring $\Lambda$. In this section we show that they are equivalent for modules in $\bmod \Lambda / x^{r} \Lambda$ and $\mathrm{CM}_{R / x^{s} R}\left(\Lambda / x^{s} \Lambda\right)$ if the numbers $t_{x}=\left|\mathrm{TF}_{x}(\Lambda)\right|_{x}$ and $c_{x}=\left|\mathrm{CM}_{R}(\Lambda)\right|_{x}$ are finite and $r \geq t_{x}+1$ and $s \geq c_{x}+1$ for the general case and the Gorenstein case, respectively. Moreover, every lifting of a liftable module in these situations is unique up to isomorphism. We also show that we have a partial converse to this result, namely if every lifting of a liftable module in $\bmod \Lambda / x^{n} \Lambda\left(\right.$ or $\left.\operatorname{CM}_{R / x^{n} R}\left(\Lambda / x^{n} \Lambda\right)\right)$ is unique up to isomorphism, then $t_{x}$ (or $c_{x}$ ) is finite and less than or equal to $n$.

Proposition 2.1 (a) Let $\Lambda, R$ and $x$ be as in the general case. Denote by $t_{x}$ the number $\left|\mathrm{TF}_{x}(\Lambda)\right|_{x}$ and assume that $t_{x}$ is finite. If $r \geq t_{x}+1$, then a module $C$ in $\bmod \Lambda / x^{r} \Lambda$ is weakly liftable to $\Lambda$ if and only if $C$ is liftable to $\Lambda$. Moreover, every lifting of a $\Lambda$-liftable module in $\bmod \Lambda / x^{r} \Lambda$ is unique up to isomorphism.

(b) Let $\Lambda, R$ and $x$ be as in the Gorenstein case. Denote by $c_{x}$ the number $\left|\mathrm{CM}_{R}(\Lambda)\right|_{x}$ and assume that $c_{x}$ is finite. If $s \geq c_{x}+1$, then a module $C$ in $\mathrm{CM}_{R / x^{s} R}\left(\Lambda / x^{s} \Lambda\right)$ is weakly liftable to $\Lambda$ if and only if $C$ is liftable to $\Lambda$. Moreover, every lifting of a $\Lambda$-liftable module in $\mathrm{CM}_{R / x^{s} R}\left(\Lambda / x^{s} \Lambda\right)$ is unique up to isomorphism.

Proof: We only give the proof for (a) since the proof of (b) is similar. Assume that $C$ in $\bmod \Lambda / x^{r} \Lambda$ is weakly liftable to $\Lambda$ with weak lifting $L$ in $\bmod \Lambda$. Then $L / x^{r} L \simeq C \oplus C^{\prime}$ for some $C^{\prime}$ in $\bmod \Lambda / x^{r} \Lambda$. By Theorem 1.3 (c) the module $L$ must decompose and the decomposition over $\Lambda / x^{r} \Lambda$ must be the same as over $\Lambda$, hence $C$ is liftable to $\Lambda$. Moreover, by Theorem 1.3 (a) the lifting is unique up to isomorphism. Since liftability always implies weak liftability, this completes the proof.

After this result it is very tempting to try to prove the converse, namely if every liftable module in $\bmod \Lambda / x^{r} \Lambda$ has a unique lifting and weak liftability is equivalent to liftability, then $r \geq t_{x}+1$. But this is not true in general and we give examples to show that almost anything can happen when $r=t_{x}$. We only have the following semi-converse, which was essentially proved in [3] and we include the proof for completeness. 
Proposition 2.2 (a) Let $\Lambda, R$ and $x$ be as in the general case. Denote by $t_{x}$ the number $\left|\mathrm{TF}_{x}(\Lambda)\right|_{x}$. If every lifting of a $\Lambda$-liftable module in $\bmod \Lambda / x^{r} \Lambda$ is unique up to isomorphism, then $r \geq t_{x}$ and in particular $t_{x}$ is finite.

(b) Let $\Lambda, R$ and $x$ be as in the Gorenstein case. Denote by $c_{x}$ the number $\left|\mathrm{CM}_{R}(\Lambda)\right|_{x}$. If every lifting of a $\Lambda$-liftable module in $\mathrm{CM}_{R / x^{s} R}\left(\Lambda / x^{s} \Lambda\right)$ is unique up to isomorphism, then $s \geq c_{x}$ and in particular $c_{x}$ is finite.

Proof: We only prove (a), since the proof of (b) is similar. Assume that every $\Lambda$-liftable $\Lambda / x^{r} \Lambda$-module has a unique lifting. Let $L$ be in $\operatorname{TF}_{x}(\Lambda)$ and let $M=L / x^{r} L$. Since $M$ is liftable to $\Lambda$, in particular to $\Lambda / x^{2 r} \Lambda$, we have that $\Omega_{\Lambda}(M) / x^{r} \Omega_{\Lambda}(M) \simeq M \oplus \Omega_{\Lambda / x^{r} \Lambda}(M)$ by [3, Prop. 1.5]. By [3, Prop. 2.4] we have that $L \oplus \Omega_{\Lambda}(L)$ is a lifting of $\Omega_{\Lambda}(M) / x^{r} \Omega_{\Lambda}(M)$ to $\Lambda$. Since $\Omega_{\Lambda}(M)$ clearly is a lifting of $\Omega_{\Lambda}(M) / x^{r} \Omega_{\Lambda}(M)$ and every lifting is unique up to isomorphism, $\Omega_{\Lambda}(M) \simeq L \oplus \Omega_{\Lambda}(L)$. By Proposition 1.1 this is equivalent to that $x^{r} \cdot \operatorname{Ext}_{\Lambda}^{1}(L)=,(0)$ and it follows that $r \geq t_{x}$.

Remark: (1) Let $\Lambda, R$ and $x$ be as in the general case and assume in addition that $R$ is contained in $\Lambda$. Denote by $t_{x}$ the number $\left|\mathrm{TF}_{x}(\Lambda)\right|_{x}$. If every $\Lambda$-liftable module in $\bmod \Lambda / x^{r} \Lambda$ has a unique lifting up to isomorphism for some $r$, then $t_{x}$ must be finite by the above proposition and by the remark after Proposition $1.6 R$ must be of dimension 1 . So, it follows immediately from the above proposition that if $\operatorname{dim} R \geq 2$, then not every $\Lambda$-lifting of a liftable module in $\bmod \Lambda / x^{r} \Lambda$ for any $r$ is unique up to isomorphism.

(2) Let $\Lambda=k[[x, y]] /\left(x^{3}+y^{2}\right)$ and let $R=k[[x]]$ be contained in $\Lambda$ in the natural way. Then $\Lambda$ is an $R$-algebra such that $\Lambda$ is a maximal CohenMacaulay module over $R$ and $\operatorname{Hom}_{R}\left(\Lambda^{\mathrm{op}}, R\right)$ and $\Lambda$ are isomorphic as left $\Lambda$-modules. Then $x$ in $R$ is a $\Lambda$-regular element and $\operatorname{TF}_{x}(\Lambda)=\mathrm{CM}_{R}(\Lambda)$. All the indecomposable nonprojective maximal Cohen-Macaulay modules over $\Lambda$ is given by $M_{r}=\Lambda^{2} / \operatorname{Im}\left(\begin{array}{cc}x^{r} & -y \\ y & x^{3-r}\end{array}\right)$ for $r=1,2$. It follows that every liftable module in $\Lambda / x \Lambda$ must have two generators, and one can show that $n_{x}\left(M_{r}\right)=1$ for $r=1,2$ and therefore $t_{x}=c_{x}=1$. Since $M_{r} / x M_{r} \simeq(\Lambda /(x, y) \Lambda)^{2} \simeq k^{2}$, the module $k$ is weakly liftable, but not liftable since $\Lambda$ is not a discrete valuation ring (By example (2) after [3, Proposition 3.2]). Moreover, the liftable $\Lambda / x \Lambda$ module $(\Lambda /(x, y) \Lambda)^{2}$ does not have a unique $\Lambda$-lifting up to isomorphism. This shows that every thing can go wrong for $r=t_{x}$. It also shows that even if $L$ is an indecomposable module in $\mathrm{CM}_{R}(\Lambda)$, then $L / x^{c_{x}} L$ is not necessarily indecomposable. This shows that the statement [12, Theorem 1.2 (c)] is not true in general.

(3) Let $R=\hat{\mathbf{Z}}_{(3)}$ be the completed ring of 3-adic integers and $G$ a cyclic group of order 3 . Then $\Lambda=R G \simeq \hat{\mathbf{Z}}_{(3)}[t] /\left(t^{3}-1\right)$, the element $x=3$ in the maximal ideal of $R$ is $\Lambda$-regular and $t_{3}=c_{3}=1$. The only indecomposable non- 
projective modules in $\mathrm{TF}_{3}(\Lambda)$ are $M_{1}=\hat{\mathbf{Z}}_{(3)}[t] /(t-1)$ and $M_{2}=\hat{\mathbf{Z}}_{(3)}[t] /\left(t^{2}+t+\right.$ $1)$. We have $\Lambda / 3 \Lambda \simeq \mathbf{Z}_{3}[t] /(t-1)^{3}$ and all the indecomposable non-projective $\Lambda / 3 \Lambda$-modules are $S_{i}=\mathbf{Z}_{3}[t] /(t-1)^{i}$ for $i=1,2$. Then it is obvious that $S_{i}$ for $i=1,2$ both have a unique $\Lambda$-lifting up to isomorphism and that weak liftability is equivalent to liftability. This shows that every liftable $\Lambda / x^{r} \Lambda$ module has a unique lifting up to isomorphism and weak liftability is equivalent liftability even for $r=t_{x}$.

(4) Let $S=\hat{\mathbf{Z}}_{(2)}$ be the completed ring of 2-adic integers and $G$ a cyclic group of order 2 . Then $\Lambda=S G \simeq \hat{\mathbf{Z}}_{(2)}[t] /\left(t^{2}-1\right)$, the element $x=2$ in the maximal ideal of $S$ is $\Lambda$-regular and $t_{2}=c_{2}=1$. It is easy to see that in this example a $\Lambda / 2 \Lambda$-module is weak $\Lambda$-liftable if and only if it is $\Lambda$-liftable, but not every liftable $\Lambda / 2 \Lambda$-module has a unique $\Lambda$-lifting up to isomorphism.

\section{Multiplication maps.}

Let $\Lambda$ be a noetherian $R$-algebra where $R$ is a complete local ring with maximal ideal $\underline{m}$. In the previous sections we have seen that elements $x$ in $\underline{m}$ regular on $\Lambda$ with the property that $x \cdot \operatorname{Ext}_{\Lambda}^{1}(M)=,(0)$ for $\Lambda$-modules $M$ on which $x$ is regular plays an important role. This section is devoted to show that if $x$ is in the center of $\Lambda$ and $x \cdot \operatorname{Ext}_{\Lambda}^{1}(M)=,(0)$ for $M$ in $\bmod \Lambda$, then $x$ is in the annihilator of the $\Lambda$-module $I_{M} / I_{M}^{2}$, where $I_{M}$ is the Fitting ideal for $M$ (see below for the definition of Fitting ideal).

Let $\Lambda$ and $R$ be as above. We start by defining the Fitting ideal for any $M$ in $\bmod \Lambda$. Let

$$
0 \rightarrow K \stackrel{g}{\rightarrow} P \stackrel{f}{\rightarrow} M \rightarrow 0
$$

be a projective cover of $M$ as a $\Lambda$-module. Then the Fitting ideal $I_{M}$ of $M$ is defined to be the ideal generated by the ideals $s \circ g(K)$ for all $s$ in $\operatorname{Hom}_{\Lambda}(P, \Lambda)$. Since multiplication by elements $\lambda$ in $\Lambda$ from the right is a (left) endomorphism of $\Lambda$, it follows that $I_{M}$ is a two-sided ideal in $\Lambda$. It is easy to see that the definition of $I_{M}$ is independent of the choice of projective cover of $M$ and since $\operatorname{Im} g \subset(\operatorname{rad} \Lambda) P$, the ideal $I_{M}$ is always a proper ideal of $\Lambda$.

Proposition 3.1 Let $\Lambda$ and $R$ be as above and let $x$ be an element in the center of $\Lambda$. If the map given by multiplication by $x, x \cdot \operatorname{id}_{M}: M \rightarrow M$, for a module $M$ in $\bmod \Lambda$ factors through a projective $\Lambda$-module, then $x$ is in the annihilator of the $\Lambda$-module $I_{M} / I_{M}^{2}$.

Proof: Let $M$ be in $\bmod \Lambda$ and let $0 \rightarrow K \stackrel{g}{\rightarrow} P \stackrel{f}{\rightarrow} M \rightarrow 0$ be a projective cover of $M$. Let $x$ be in the center of $\Lambda$ and assume that the map given by 
multiplication by $x, x \cdot \mathrm{id}_{M}: M \rightarrow M$, factors through a projective $\Lambda$-module. Then the map $x \cdot \operatorname{id}_{P}: M \rightarrow M$ factors in particular through $f$ and we have the following diagram,

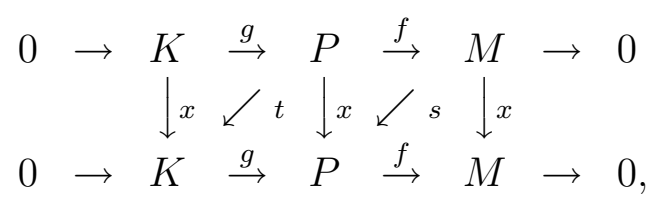

where $f_{\circ} s=x \cdot \operatorname{id}_{M}$ and $x \cdot \operatorname{id}_{M}=g \circ t+s \circ f$. Applying $g$ from the right on this equation gives the equation $x \cdot g=g \circ t \circ g$, since $f \circ g=0$. Let $\pi=$ $\left(\pi_{i}\right)_{i=1}^{n}: \Lambda^{n} \rightarrow K$ be an epimorphism. Then since $P$ is projective, there exists a map $\alpha=\left(\alpha_{i}\right)_{i=1}^{n}: P \rightarrow \Lambda^{n}$ such that $t=\pi_{\circ} \alpha=\sum_{i=1}^{n} \pi_{i} \circ \alpha_{i}$. Let $\lambda$ be any element in $\operatorname{Hom}_{\Lambda}(P, \Lambda)$ and $a$ any element in $K$. Then we have that

$$
\begin{aligned}
x \cdot \lambda \circ g(a) & =\lambda \circ g \circ t \circ g(a) \\
& =\sum_{i=1}^{n} \lambda \circ g \circ \pi_{i}\left(\alpha_{i}(g(a))\right) \\
& =\sum_{i=1}^{n}\left(\alpha_{i}(g(a))\right) \cdot \lambda \circ g \circ \pi_{i}(1), \text { where } \pi_{i}(1) \in K .
\end{aligned}
$$

Since $\alpha_{i}$ is in $\operatorname{Hom}_{\Lambda}(P, \Lambda)$ for all $i$, it follows that $\alpha_{i}(g(a))$ is in $I_{M}$, hence $\sum_{i=1}^{n}\left(\alpha_{i}(g(a))\right) \cdot \lambda \circ g\left(\pi_{i}(1)\right)$ is in $I_{M}^{2}$. Therefore $x \cdot \lambda \circ g(a)$ is in $I_{M}^{2}$ and $x$ is in the annihilator of the $\Lambda$-module $I_{M} / I_{M}^{2}$.

The Fitting ideal $I_{M}$ is contained in the annihilator of $I_{M} / I_{M}^{2}$. By the above proposition the ideal $\beta(M)=\left\{\lambda \in \Lambda \cap Z(\Lambda) \mid \lambda \cdot \operatorname{Ext}_{\Lambda}^{1}(M)=,(0)\right\}$ is also contained in the annihilator of $I_{M} / I_{M}^{2}$. But there is no inclusion relation between $I_{M} \cap Z(\Lambda)$ and $\beta(M)$ in general. The following examples illustrate this.

Examples: (1) Let $R=\hat{\mathbf{Z}}_{(3)}$ be the completed ring of 3-adic integers and $G$ a cyclic group of order 27 . Then $\Lambda=R G \simeq \hat{\mathbf{Z}}_{(3)}[t] /\left(t^{27}-1\right)$ and $t^{27}-1=\left(t^{3}-1\right)\left(\sum_{i=0}^{8} t^{3 i}\right)$. Let $Q=\sum_{i=0}^{8} t^{3 i}$ and $M=\hat{\mathbf{Z}}_{(3)}[t] /\left(t^{3}-1\right)$, which has the following presentation

$$
0 \rightarrow\left(t^{3}-1\right) /\left(t^{27}-1\right) \rightarrow \Lambda \rightarrow M \rightarrow 0 .
$$

Therefore $I_{M}=\left(t^{3}-1\right) /\left(t^{27}-1\right)$ and $\operatorname{ann}_{\Lambda} I_{M} / I_{M}^{2}=\left(t^{3}-1, Q\right) /\left(t^{27}-1\right)$. Since $\operatorname{Hom}_{\Lambda}(M, \Lambda)=\Lambda Q$ and the projective cover of $M$ is $\Lambda$, the multiplication map given by $Q, Q \cdot \operatorname{id}_{M}: M \rightarrow M$, factors through a projective. Since $\left(t^{3}-\right.$ 1) $\cdot \operatorname{id}_{M}: M \rightarrow M$ is the zero map and $\beta(M) \subset$ ann ${ }_{\Lambda} I_{M} / I_{M}^{2}$, it follows that $\beta(M)=\operatorname{ann}_{\Lambda} I_{M} / I_{M}^{2}$. Therefore $I_{M} \cap Z(\Lambda)$ is a proper subset of $\beta(M)$.

(2) Let $\Lambda=k[[x, y]] /\left(y^{3}+x^{4}\right)$, where $k$ is an algebraically closed field of characteristic different from two. The ring $\Lambda$ is a complete commutative local 
Gorenstein ring of dimension 1 , the $\mathrm{E}_{6}$-singularity in dimension 1. Let

$$
\phi=\left(\begin{array}{ccc}
y & 0 & x \\
x & y & 0 \\
0 & x^{2} & y
\end{array}\right): \Lambda^{3} \rightarrow \Lambda^{3} .
$$

Then $M=\Lambda^{3} / \operatorname{Im} \phi$ is a maximal Cohen-Macaulay module over $\Lambda$ and $I_{M}=$ $(x, y)$. One can show that $\beta(M)=\left(x^{2}, x y, y^{2}\right)$, hence $\beta(M)$ is a proper subset of $I_{M} \cap Z(\Lambda)$.

\section{Examples.}

Let $A=k\left[\left[z_{0}, \ldots, z_{d}\right]\right]$ be the formal power series ring in the variables $z_{i}$ over an algebraically closed field $k$ of characteristic 0 . This last section is devoted to calculating the numbers $n_{x}()$ and $c_{x}$ for the simple isolated singularities $\mathrm{A}_{n}, \mathrm{D}_{n}, \mathrm{E}_{6}, \mathrm{E}_{7}$ and $\mathrm{E}_{8}$ in $A$ in any dimension.

Let $A$ be as above. The simple isolated singularities $\mathrm{A}_{n}, \mathrm{D}_{n}, \mathrm{E}_{6}, \mathrm{E}_{7}$ and $\mathrm{E}_{8}$ are given by the following equations

$$
\begin{array}{rll}
A_{n}: & z_{0}^{n+1}+z_{1}^{2}+\cdots+z_{d}^{2} & (n \geq 1) \\
D_{n}: & z_{0}^{2} z_{1}+z_{1}^{n-1}+z_{2}^{2}+\cdots+z_{d}^{2} \quad(n \geq 4) \\
E_{6}: & z_{0}^{4}+z_{1}^{3}+z_{2}^{2}+\cdots+z_{d}^{2} & \\
E_{7}: & z_{0}^{3}+z_{0} z_{1}^{3}+z_{2}^{2}+\cdots+z_{d}^{2} & \\
E_{8}: & z_{0}^{5}+z_{1}^{3}+z_{2}^{2}+\cdots+z_{d}^{2} . &
\end{array}
$$

Let $R=A /(f)$ where $f$ is a nonzero nonunit in $A$ and let $\operatorname{CM}(R)$ denote the category of all maximal Cohen-Macaulay modules over $R$. Then it was shown in [8] that $\operatorname{CM}(R)$ and $\operatorname{CM}\left(A[[x, y]] /\left(f+x^{2}+y^{2}\right)\right)$ are stably equivalent. Using this it turns out that it is sufficient to calculate the numbers $n_{x}()$ and $c_{x}$ for dimension 1 and 2 .

Before we can start calculating the numbers $n_{x}()$ and $c_{x}$, we need some preliminary results and notation. Let $A, R$ and $f$ be as above. We recall from [6] that a matrix factorization $(\phi, \psi)$ of $f$ in $A$ over $A$ is a pair of $A$ homomorphisms, $\phi: P \rightarrow Q$ and $\psi: Q \rightarrow P$ between finitely generated free $A$-modules $P$ and $Q$ such that $\phi \circ \psi=f \cdot \mathrm{id}_{Q}$ and $\psi_{\circ} \phi=f \cdot \mathrm{id}_{P}$. A morphism $(\alpha, \beta):(\phi, \psi) \rightarrow\left(\phi^{\prime}, \psi^{\prime}\right)$ is a pair of $A$-homomorphisms such that the following diagram commutes

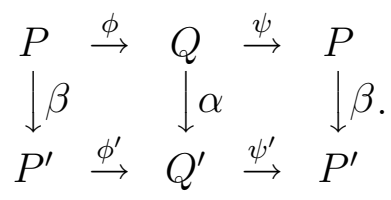


Denote by $\mathrm{MF}_{A}(f)$ the category of all matrix factorizations of $f$ over $A$. Define cok: $\mathrm{MF}_{A}(f) \rightarrow \mathrm{CM}(R)$ by $\operatorname{cok}(\phi, \psi)=\operatorname{coker} \phi$ and $\operatorname{cok}(\alpha, \beta)=\bar{\alpha}$ for $(\alpha, \beta):(\phi, \psi) \rightarrow\left(\phi^{\prime}, \psi^{\prime}\right)$, where $\bar{\alpha}$ denotes the homomorphism $\alpha$ induces between coker $\phi$ and coker $\phi^{\prime}$. This functor is exact and $\mathrm{MF}_{A}(f) /(1, f)$ is equivalent to $\operatorname{CM}(R)[6,8]$, where $\mathrm{MF}_{A}(f) /(1, f)$ is the category $\mathrm{MF}_{A}(f)$ modulo the morphisms factoring through copies of $(1, f)$. The objects $(f, 1)$ and $(1, f)$ are the only indecomposable projective objects in $\mathrm{MF}_{A}(f)$. Given $(\phi, \psi)$ and $\left(\phi^{\prime}, \psi^{\prime}\right)$ in $\mathrm{MF}_{A}(f)$ the morphisms factoring through copies of $(f, 1)$ and $(1, f)$ are of the form $\left(K \psi, \psi^{\prime} K\right)$ and $\left(\phi^{\prime} L, L \phi\right)$, respectively, where $K$ and $L$ are arbitrary matrices of suitable sizes [11, Lemma 3.2]. Let $M$ be in $\operatorname{CM}(R)$ and let $\left(\phi: A^{n} \rightarrow A^{n}, \psi: A^{n} \rightarrow A^{n}\right)$ be a corresponding matrix factorization, since the rank of $P$ and $Q$ are equal by [6, Corollary 5.4]. From the above it follows easily that a multiplication map $x \cdot \operatorname{id}_{M}: M \rightarrow M$ factors through a projective if and only if there exists an $n \times n$-matrix $K$ such that for all $a$ in $A^{n}$ there exists $a^{\prime}$ in $A^{n}$ such that $K \psi(a)+\phi\left(a^{\prime}\right)=x \cdot a$.

As in [8] let the functor $F: \mathrm{MF}_{A}(f) \rightarrow \mathrm{MF}_{B}\left(f+x^{2}+y^{2}\right)$ be given by

$$
F((\phi, \psi))=\left(\left(\begin{array}{cc}
(x+i y) \mathrm{I}_{n} & \phi \\
\psi & (-x+i y) \mathrm{I}_{n}
\end{array}\right),\left(\begin{array}{cc}
(x-i y) \mathrm{I}_{n} & \phi \\
\psi & (-x-i y) \mathrm{I}_{n}
\end{array}\right)\right)
$$

for all $(\phi, \psi)$ in $\mathrm{MF}_{A}(f)$, where $\phi$ and $\psi$ are $n \times n$-matrices and $B=A[[x, y]]$. On morphisms $(\alpha, \beta):(\phi, \psi) \rightarrow\left(\phi^{\prime}, \psi^{\prime}\right)$ in $\mathrm{MF}_{A}(f)$, the functor $F$ is given by

$$
F((\alpha, \beta))=\left(\left(\begin{array}{cc}
\alpha & 0 \\
0 & \beta
\end{array}\right),\left(\begin{array}{cc}
\alpha & 0 \\
0 & \beta
\end{array}\right)\right) .
$$

In [8] $F$ is shown to induce an equivalence between $\mathrm{MF}_{A}(f)$ and $\mathrm{MF}_{B}(f+$ $\left.x^{2}+y^{2}\right)$ modulo the projective objects. Let $M$ be in $\mathrm{CM}(R)$ with a corresponding matrix factorization $(\phi, \psi)$ and let $\tilde{\beta}_{A}(M)=\left\{t \in A \mid t \cdot \operatorname{id}_{M}: M \rightarrow\right.$ $M$ factors through a projective $\}$. The above suggests that there is a strong connection between $\tilde{\beta}_{A}(M)$ and $\tilde{\beta}_{B}\left(\operatorname{cok}_{B}(F((\phi, \psi)))\right)$. In fact we have the following connection.

Lemma 4.1 Let $M$ be in $\mathrm{CM}(R)$ with a corresponding matrix factorization $(\phi, \psi)$. Then $\tilde{\beta}_{B}\left(\operatorname{cok}_{B}(F((\phi, \psi)))\right)=\left(\tilde{\beta}_{A}(M), x, y\right)$.

Proof: Let $A, B, f$ and $R$ be as above. Denote $B /\left(f+x^{2}+y^{2}\right)$ by $S$. Let $M$ be a module in $\operatorname{CM}(R)$ with a corresponding matrix factorization $(\phi, \psi)$, where $\phi$ and $\psi$ are $n \times n$ matrices. Assume that $t \cdot \operatorname{id}_{M}: M \rightarrow M$ factors through a projective module with $t$ in $R$. Let $\tilde{t}$ be in $A$ such that $\tilde{t}=t$ in $R$. Since $\operatorname{cok}_{A}: \mathrm{MF}_{A}(f) /(1, f) \rightarrow \mathrm{CM}(R)$ is an equivalence, there exist maps $u:(\phi, \psi) \rightarrow(f, 1)^{r} \oplus(1, f)^{s}$ and $v:(f, 1)^{r} \oplus(1, f)^{s} \rightarrow(\phi, \psi)$ such that $v \circ u=$ 
$\tilde{t} \cdot \operatorname{id}_{(\phi, \psi)}$. Applying the functor $F$ to these maps we get maps $u^{\prime}: F(\phi, \psi) \rightarrow$ $\left(f+x^{2}+y^{2}, 1\right)^{r+s} \oplus\left(1, f+x^{2}+y^{2}\right)^{r+s}$ and $v^{\prime}:\left(f+x^{2}+y^{2}, 1\right)^{r+s} \oplus(1, f+$ $\left.x^{2}+y^{2}\right)^{r+s} \rightarrow F(\phi, \psi)$, such that $v^{\prime} \circ u^{\prime}=\tilde{t} \cdot \operatorname{id}_{F(\phi, \psi)}$. After applying the functor $\operatorname{cok}_{B}: \mathrm{MF}_{B}\left(f+x^{2}+y^{2}\right) \rightarrow C M(S)$ we see that $\tilde{t} \cdot \operatorname{id}_{\operatorname{cok}_{B} F(\phi, \psi)}$ factors through $S^{r+s}$. This shows that $\tilde{\beta}_{A}(M) B \subset \tilde{\beta}_{B}\left(\operatorname{cok}_{B} F(\phi, \psi)\right)$. On the other hand assume that $t \cdot \mathrm{id}_{\operatorname{cok}_{B} F(\phi, \psi)}$ for a $t$ in $B$ factors through a projective. Let $G=$ $S /(x, y) S \otimes_{S^{-}}: \operatorname{CM}(S) \rightarrow \operatorname{CM}(R)$. Then it is easy to see that $G \circ{ }_{\circ} \operatorname{cok}_{B} F(\phi, \psi) \simeq$ $M \oplus \Omega_{R}(M)$. Let $\bar{t}$ denote $t$ as an element in $S /(x, y) S \simeq R$. It follows that $\bar{t} \cdot \operatorname{id}_{G \circ \text { ocok }_{B} F(\phi, \psi)}: M \oplus \Omega_{R}(M) \rightarrow M \oplus \Omega_{R}(M)$ factors through a projective $R$ module. Therefore $\bar{t} \cdot \mathrm{id}_{M}$ also factors through a projective $R$-module. This shows that $\tilde{\beta}_{B}\left(\operatorname{cok}_{B} F(\phi, \psi)\right)$ viewed as elements in $S /(x, y) S \simeq R$ is contained in $\tilde{\beta}_{A}(M)$.

Assume that we have shown that $x$ and $y$ are elements in $\tilde{\beta}_{B}\left(\operatorname{cok}_{B} F(\phi, \psi)\right)$. Then our desired result follows from what we already have proved.

We want to prove that $x \cdot \operatorname{id}_{\operatorname{cok}_{B} F(\phi, \psi)}$ factors through a projective module. Using the above remark the map $x \cdot \mathrm{id}_{\operatorname{cok}_{B} F(\phi, \psi)}$ factors through a projective module if and only if there exists a matrix $K$ in $M_{2 n}(S)$ such that for every $\left(s_{1}, s_{2}\right)$ in $S^{n} \oplus S^{n}$ there exists $s^{\prime}$ in $S^{n} \oplus S^{n}$ such that

$$
K\left(\begin{array}{cc}
\psi & -x \\
y & \phi
\end{array}\right)\left(\begin{array}{l}
s_{1} \\
s_{2}
\end{array}\right)+\left(\begin{array}{cc}
\phi & x \\
-y & \psi
\end{array}\right) s^{\prime}=x \cdot\left(\begin{array}{l}
s_{1} \\
s_{2}
\end{array}\right)
$$

Let $K=\left(\begin{array}{cc}0 & 0 \\ -\mathrm{I}_{n} & 0\end{array}\right)$ and $s^{\prime}=\left(0, s_{1}\right)$. Then the above equation is satisfied. Hence $x$ and by symmetry $y$ are elements in $\tilde{\beta}_{B}\left(\operatorname{cok}_{B} F(\phi, \psi)\right)$.

So, for the above singularities we just have to calculate the numbers for dimension 1 and 2 , since $n_{t}(M)$ is the smallest nonzero natural number $m$ such that $t^{m}$ is in $\beta_{R}(M)=\left\{r \in R \mid r \cdot \operatorname{id}_{M}: M \rightarrow M\right.$ factors through a projective $\}$, which is equal to $\tilde{\beta}_{A}(M) /(f)$. Let us first consider the $\mathrm{A}_{n}$-singularity in dimension 1 .

The $\mathrm{A}_{n}$-singularity in dimension 1 in $A=k\left[\left[z_{0}, z_{1}\right]\right]$ is given by $f=z_{0}^{n+1}+z_{1}^{2}$ and the corresponding local ring is $R=A /(f)$. Then by [13, Proposition 5.11 and 9.9] all the indecomposable maximal Cohen-Macaulay modules over $R$ are given by: $R$ and $M_{r}=A^{2} / \operatorname{Im}\left(\begin{array}{cc}z_{0}^{r} & -z_{1} \\ z_{1} & z_{0}^{n+1-r}\end{array}\right)$ for $r=1,2, \ldots,\left[\frac{n+1}{2}\right]$ and if $n=$ $2 m-1$, then instead of $M_{m}$ we have $M_{+}=A /\left(z_{1}+i z_{0}^{m}\right)$ and $M_{-}=A /\left(z_{1}-i z_{0}^{m}\right)$. The corresponding matrix factorization for $M_{r}$ is $\left(\left(\begin{array}{cc}z_{0}^{r} & -z_{1} \\ z_{1} & z_{0}^{n+1-r}\end{array}\right),\left(\begin{array}{cc}z_{0}^{n+1-r} & z_{1} \\ -z_{1} & z_{0}^{r}\end{array}\right)\right)=$ $\left(\phi_{r}, \psi_{r}\right)$. Then as before a multiplication map $t \cdot \operatorname{id}_{M_{r}}: M_{r} \rightarrow M_{r}$ with $t$ in $A$ factors through a projective module if and only if there exists a matrix $K$ in 
$M_{2}(A)$ such that for every $\left(s_{1}, s_{2}\right)$ in $A^{2}$ there exists $s^{\prime}$ in $A^{2}$ such that

$$
K \psi_{r}\left(\begin{array}{l}
s_{1} \\
s_{2}
\end{array}\right)+\phi_{r}\left(s^{\prime}\right)=t \cdot\left(\begin{array}{l}
s_{1} \\
s_{2}
\end{array}\right) .
$$

Let $K=\left(\begin{array}{ll}a & b \\ c & d\end{array}\right)$. If $t=z_{1}$, then choose $b=-1, a=c=d=0$ and $s^{\prime}=\left(s_{2}, 0\right)$. Since $z_{1}$ is in $\beta_{R}\left(M_{r}\right)$, it is enough to determined what power of $z_{0}$ that is in $\beta_{R}\left(M_{r}\right)$ to find the ideal $\beta_{R}\left(M_{r}\right)$. When $t=z_{0}^{r}$, choose $d=1, a=b=c=0$ and $s^{\prime}=\left(0, s_{2}\right)$. For $t=z_{0}^{n+1-r}$, let $a=1, b=c=d=0$ and $s^{\prime}=\left(0, s_{2}\right)$. Let $t=z_{0}^{v}$ with $v<\min \{r, n+1-r\}$. If the above equation is satisfied for $t$, then $z_{0}^{v}$ is in the ideal $\left(z_{0}^{r}, z_{0}^{n+1-r}, z_{1}\right)$. This is impossible, hence $\beta_{R}\left(M_{r}\right)=\left(z_{0}^{r}, z_{1}\right)$ for $r=1,2, \ldots,\left[\frac{n+1}{2}\right]$.

It remains to discuss the case $n=2 m-1$ and the modules $M_{+}$and $M_{-}$. Assume that $t$ is in $\beta_{R}\left(M_{+}\right)$. By Proposition 3.1 the element $t$ is in ann ${ }_{R} I_{M_{+}} / I_{M_{+}}^{2}$, where $I_{M_{+}}$is the Fitting ideal of $M_{+}$. We have that $I_{M_{+}}=\left(z_{1}+i z_{0}^{m}\right) /\left(z_{0}^{n}+z_{1}^{2}\right)$, so that ann ${ }_{R} I_{M_{+}} / I_{M_{+}}^{2}=\left(z_{1}+i z_{0}^{m}, z_{1}-i z_{0}^{m}\right)=\left(z_{1}, z_{0}^{m}\right)$. The multiplication $\operatorname{map}\left(z_{1}+i z_{0}^{m}\right) \cdot \operatorname{id}_{M_{+}}$is the zero map, hence $z_{1}+i z_{0}^{m}$ is in $\beta_{R}\left(M_{+}\right)$. The composition of the maps $\left(z_{1}-i z_{0}^{m}\right): M_{+} \rightarrow R$ and the natural projection map $R \rightarrow M_{+}$is the multiplication map $\left(z_{1}+i z_{0}^{m}\right) \cdot \mathrm{id}_{M_{+}}$. This shows that $\beta_{R}\left(M_{+}\right)=\operatorname{ann}_{R} I_{M_{+}} / I_{M_{+}}^{2}=\left(z_{1}, z_{0}^{m}\right)$. Since $M_{-} \simeq \Omega_{R}\left(M_{+}\right)$, we have by Corollary 1.8 that $\beta_{R}\left(M_{+}\right)=\beta_{R}\left(M_{-}\right)=\left(z_{1}, z_{0}^{m}\right)$.

The remark after Lemma 4.1 gives how to calculate $n_{t}(M)$ for any $t$ in the maximal ideal $\underline{m}$ of $R$ and any $M$ in $\operatorname{CM}(R)$. In particular we have $n_{z_{0}}\left(M_{r}\right)=r$ for $r=1,2, \ldots,\left[\frac{n+1}{2}\right]$ and if $n=2 m-1$, then $n_{z_{0}}\left(M_{+}\right)=n_{z_{0}}\left(M_{-}\right)=m=\left[\frac{n+1}{2}\right]$. We also have $n_{z_{1}}(M)=1$ for any nonprojective indecomposable module $M$ in $\mathrm{CM}(R)$ and moreover $n_{t}(M) \leq\left[\frac{n+1}{2}\right]$ for any $t$ in $\underline{m}$ and any $M$ in $\operatorname{CM}(R)$.

The $\mathrm{A}_{n}$-singularity in dimension 2 in $A=k\left[\left[z_{0}, z_{1}, z_{2}\right]\right]$ is given by $f=$ $z_{0}^{n+1}+z_{1}^{2}+z_{2}^{2}$ and the corresponding local ring is $R=A /(f)$. Using that $\operatorname{CM}(R)$ and $\operatorname{CM}\left(k\left[\left[z_{0}\right]\right] /\left(z_{0}^{n+1}\right)\right)$ are stably equivalent induced by the functor above, it follows that $R$ and $M_{r}=A^{2} / \operatorname{Im}\left(\begin{array}{cc}z_{1}+i z_{2} & z_{0}^{r} \\ z_{0}^{n+1-r}-z_{1}+i z_{2}\end{array}\right)$ for $r=1,2, \ldots, n$ are all the indecomposable maximal Cohen-Macaulay modules over $R$. By applying Lemma 4.1 it is easily verified that $\beta_{R}\left(M_{r}\right)=\left(z_{0}^{m}, z_{1}, z_{2}\right)$, where $m=\min \{r, n+1-r\}$. In particular it follows that $n_{z_{0}}\left(M_{r}\right)=\min \{r, n+1-r\}$ and $n_{z_{i}}\left(M_{r}\right)=1$ for all $r=1,2, \ldots, n$ and $i=1,2$ and moreover $n_{t}(M) \leq\left[\frac{n+1}{2}\right]$ for any $t$ in $\underline{m}$ and any $M$ in $\operatorname{CM}(R)$.

Proposition 4.2 Let $A=k\left[\left[z_{0}, z_{1}, \ldots, z_{d}\right]\right], f=z_{0}^{n+1}+z_{1}^{2}+\cdots+z_{d}^{2}$ and $R=A /(f)$, the $A_{n}$-singularity in dimension d. Then $|\mathrm{CM}(R)|_{z_{0}}=\left[\frac{n+1}{2}\right]$ and $|\mathrm{CM}(R)|_{z_{i}}=1$ for $i=1,2, \ldots, d$. Moreover $|\mathrm{CM}(R)|_{t} \leq\left[\frac{n+1}{2}\right]$ for any $t$ in $\underline{m}$. 
Now, let us consider the $\mathrm{E}_{6}$-singularity and we start with dimension 1 . Let $A=k\left[\left[z_{0}, z_{1}\right]\right], f=z_{0}^{4}+z_{1}^{3}$ and $R=A /(f)$. By [13, 9.13] all the indecomposable maximal Cohen-Macaulay modules over $R$ are given by: $R$, $M_{1}=A^{2} / \operatorname{Im}\left(\begin{array}{cc}z_{1} & z_{0}^{2} \\ -z_{0}^{2} & z_{1}^{2}\end{array}\right), M_{2}=A^{2} / \operatorname{Im}\left(\begin{array}{cc}z_{1} & z_{0} \\ -z_{0}^{3} & z_{1}^{2}\end{array}\right), M_{3}=A^{2} / \operatorname{Im}\left(\begin{array}{cc}z_{1}^{2} & -z_{0} \\ z_{0}^{3} & z_{1}\end{array}\right), M_{4}=$ $A^{3} / \operatorname{Im}\left(\begin{array}{ccc}z_{1}^{2} & z_{0}^{3} & -z_{0} z_{1} \\ -z_{0} z_{1} & z_{1}^{2} & z_{0}^{2} \\ z_{0}^{3} & -z_{0}^{2} z_{1} & z_{1}^{2}\end{array}\right), M_{5}=A^{3} / \operatorname{Im}\left(\begin{array}{ccc}z_{1} & 0 & z_{0} \\ z_{0} & z_{1} & 0 \\ 0 & z_{0}^{2} & z_{1}\end{array}\right)$ and $M_{6}=$ $A^{4} / \operatorname{Im}\left(\begin{array}{cccc}z_{1} & z_{0}^{2} & -z_{0} & 0 \\ -z_{0}^{2} & z_{1}^{2} & 0 & z_{0} z_{1} \\ 0 & 0 & z_{1} & z_{0}^{2} \\ 0 & 0 & -z_{0}^{2} & z_{1}^{2}\end{array}\right)$. Applying the same methods as for the $\mathrm{A}_{n^{-}}$ singularities it is straightforward to see that $\beta\left(M_{1}\right)=\left(z_{0}^{2}, z_{1}\right), \beta\left(M_{2}\right)=$ $\beta\left(M_{3}\right)=\left(z_{0}, z_{1}\right), \beta\left(M_{4}\right)=\beta\left(M_{5}\right)=\beta\left(M_{6}\right)=\left(z_{0}^{2}, z_{0} z_{1}, z_{1}^{2}\right)$. Then by the remark after Lemma 4.1 the numbers $n_{t}(M)$ can be easily calculated for any $t$ in $\underline{m}$ and any $M$ in $\operatorname{CM}(R)$.

In order to study the $\mathrm{E}_{6}$-singularity and the other singularities in dimension 2 it is useful to consider the following functor between singularities $f$ and $f+z^{2}[8]$. Let $A=k\left[\left[z_{0}, \ldots, z_{d}\right]\right]$, the element $f$ a nonzero nonunit in $A$ and $R=A /(f)$. Further, let $B=A[[z]]$ and $S=B /\left(f+z^{2}\right)$. Define $F: \mathrm{MF}_{A}(f) \rightarrow$ $\mathrm{MF}_{B}\left(f+z^{2}\right)$ by

$$
F((\phi, \psi))=\left(\left(\begin{array}{cc}
\phi & z \mathrm{I}_{n} \\
-z \mathrm{I}_{n} & \psi
\end{array}\right),\left(\begin{array}{cc}
\psi & -z \mathrm{I}_{n} \\
z \mathrm{I}_{n} & \phi
\end{array}\right)\right)
$$

for all $(\phi, \psi)$ in $\mathrm{MF}_{A}(f)$, where $\phi$ and $\psi$ are $n \times n$-matrices. On morphisms $(\alpha, \beta):(\phi, \psi) \rightarrow\left(\phi^{\prime}, \psi^{\prime}\right)$ in $\mathrm{MF}_{A}(f)$, the functor $F$ is given by

$$
F((\alpha, \beta))=\left(\left(\begin{array}{cc}
\alpha & 0 \\
0 & \beta
\end{array}\right),\left(\begin{array}{cc}
\beta & 0 \\
0 & \alpha
\end{array}\right)\right) .
$$

It is shown in [8] that $F((\phi, \psi))$ is indecomposable if and only if $(\phi, \psi)$ is indecomposable and $(\phi, \psi) \not(\psi, \phi)$. Moreover every matrix factorization of $f+z^{2}$ is a summand of $F((\phi, \psi))$ for some $(\phi, \psi)$ in $\mathrm{MF}_{A}(f)$ [8, Proposition 2.7 (i) and Lemma 2.5 (ii)]. Further, if $(\phi, \psi)$ is indecomposable and $F((\phi, \psi))$ decomposes, then $F((\phi, \psi)) \simeq\left(\phi^{\prime}, \psi^{\prime}\right) \oplus\left(\psi^{\prime}, \phi^{\prime}\right)$, where $\left.\left(\phi^{\prime}, \psi^{\prime}\right) \not \psi^{\prime}, \phi^{\prime}\right)$ and $\left(\phi^{\prime}, \psi^{\prime}\right)$ is indecomposable. So given the matrix factorizations in dimension 1 and the above facts from [8], to find all indecomposable maximal Cohen-Macaulay modules over $S$ we do the following. We apply $F$ to all the indecomposable matrix factorizations of $f$ and decompose if possible and finally we apply the functor $\operatorname{cok}_{B}$ to all the indecomposable matrix factorizations we have gotten this way. 
Similar to the correspondence between $f$ and $f+x^{2}+y^{2}$ for the ideals $\tilde{\beta}$, we have the following correspondence for the ideals $\tilde{\beta}$ for the singularities $f$ and $f+z^{2}$.

Lemma 4.3 Let $A, B$ and $f$ be as above. If $M$ is an indecomposable nonprojective module in $\mathrm{CM}(R)$ and $(\phi, \psi)$ a corresponding reduced matrix factorization in $\mathrm{MF}_{A}(f)$ and if $N$ is a summand of $\operatorname{cok}_{B} F((\phi, \psi))$, then $\tilde{\beta}_{B}(N)=$ $\left(\tilde{\beta}_{A}(M), z\right)$.

Proof: Let $M^{\prime}=\operatorname{cok}_{B} F((\phi, \psi))$. Then $\left(\begin{array}{cc}z & \phi \\ 0 & 0\end{array}\right): M^{\prime} \rightarrow M^{\prime}$ is multiplication by $z$ and factors through a projective module. By Proposition 1.7 (c) multiplication by $z$ factors through a projective module for any summand of $M^{\prime}$.

Let $H: \mathrm{MF}_{B}\left(f+z^{2}\right) \rightarrow \mathrm{MF}_{A}(f)$ be the restriction functor, then $H F((\phi, \psi)) \simeq$ $(\phi, \psi) \oplus(\phi, \psi)$. Assume that $F((\phi, \psi))$ decomposes for $(\phi, \psi)$ indecomposable. Then we know from [8] that $F((\phi, \psi)) \simeq\left(\phi^{\prime}, \psi^{\prime}\right) \oplus\left(\psi^{\prime}, \phi^{\prime}\right)$, where $\left(\phi^{\prime}, \psi^{\prime}\right) \not \varkappa\left(\psi^{\prime}, \phi^{\prime}\right)$ and $\left(\phi^{\prime}, \psi^{\prime}\right)$ is indecomposable. Let $M_{1}=\operatorname{cok}_{B}\left(\phi^{\prime}, \psi^{\prime}\right)$, then $\tilde{\beta}_{B}\left(M_{1}\right)=\tilde{\beta}_{B}\left(\Omega_{S}\left(M_{1}\right)\right.$ by Corollary 1.8 and therefore also equal to $\tilde{\beta}_{B}\left(M^{\prime}\right)$. It follows that if $t \cdot \mathrm{id}_{M_{1}}$ factors through a projective module, then $t_{z=0} \cdot \mathrm{id}_{M}$ factors through a projective module, since $H\left(\phi^{\prime}, \psi^{\prime}\right)$ is isomorphic to $(\phi, \psi)$ or $(\psi, \phi)$. Conversely, if $t \cdot \mathrm{id}_{M}$ factors through a projective module, then $(t, t)$ in $\operatorname{End}_{M F_{A}(f)}((\phi, \psi))$ factors through copies of $(f, 1)$ and $(1, f)$, since $\mathrm{MF}_{A}(f) /((f, 1),(1, f))$ and $\mathrm{CM}(R) / R$ are equivalent $[6,8]$. Since the functor $F$ sends projective matrix factorizations to projective matrix factorizations, the homomorphism $t \cdot \operatorname{id}_{M_{1}}$ factors through a projective module. Therefore we have that $\tilde{\beta}_{B}\left(M_{1}\right)=\left(\tilde{\beta}_{A}(M), z\right)$.

Let $A=k\left[\left[z_{0}, z_{1}\right]\right]$ and $f=z_{0}^{4}+z_{1}^{3}$. Then $f+z_{2}^{2}$ is the $\mathrm{E}_{6}$-singularity in $B=A\left[\left[z_{2}\right]\right]$ in dimension 2. Using the above known facts from [8] all the indecomposable maximal Cohen-Macaulay modules over $S$ are: $S, M_{1}=$ $B^{2} / \operatorname{Im}\left(\begin{array}{cc}z_{1} & z_{0}^{2}+i z_{2} \\ -z_{0}^{2}+i z_{2} & z_{1}^{2}\end{array}\right)=B^{2} / \operatorname{Im} \phi, M_{2}=B^{2} / \operatorname{Im}\left(\begin{array}{cc}z_{1}^{2} & z_{0}^{2}-i z_{2} \\ -z_{0}^{2}-i z_{2} & z_{1}\end{array}\right)=B^{2} / \operatorname{Im} \psi$, $M_{3}=B^{4} / \operatorname{Im}\left(\begin{array}{cccc}z_{1} & z_{0}^{2} & z_{2} & 0 \\ -z_{0}^{2} & z_{1}^{2} & 0 & z_{2} \\ -z_{2} & 0 & z_{1}^{2} & -z_{0}^{2} \\ 0 & -z_{2} & z_{0}^{2} & z_{1}\end{array}\right), M_{4}=B^{4} / \operatorname{Im}\left(\begin{array}{ccc}\phi & -z_{0} & 0 \\ 0 & z_{0} z_{1} \\ 0 & \phi\end{array}\right), M_{5}=$ 


$$
\left.\begin{array}{rl}
B^{4} / \operatorname{Im}\left(\begin{array}{ccc}
\psi & z_{0} z_{1} & 0 \\
0 & 0 & \psi
\end{array} z_{0}\right.
\end{array}\right) \text { and }\left(\begin{array}{cccccc}
z_{1}^{2} & z_{0}^{3} & -z_{0} z_{1} & z_{2} & 0 & 0 \\
-z_{0} z_{1} & z_{1}^{2} & z_{0}^{2} & 0 & z_{2} & 0 \\
z_{0}^{3} & -z_{0}^{2} z_{1} & z_{1}^{2} & 0 & 0 & z_{2} \\
-z_{2} & 0 & 0 & z_{1} & 0 & z_{0} \\
0 & -z_{2} & 0 & z_{0} & z_{1} & 0 \\
0 & 0 & -z_{2} & 0 & z_{0}^{2} & z_{1}
\end{array}\right) .
$$

Then using Lemma 4.3 we have $\beta\left(M_{1}\right)=\beta\left(M_{2}\right)=\left(z_{0}^{2}, z_{1}, z_{2}\right), \beta\left(M_{3}\right)=$ $\left(z_{0}, z_{1}, z_{2}\right)$ and $\beta\left(M_{4}\right)=\beta\left(M_{5}\right)=\beta\left(M_{6}\right)=\left(z_{0}^{2}, z_{0} z_{1}, z_{1}^{2}, z_{2}\right)$. As before the number $n_{t}(M)$ is easily obtained from this for any $t$ in $\underline{m}$ and any $M$ in $\mathrm{CM}(S)$.

Proposition 4.4 Let $A=k\left[\left[z_{0}, z_{1}, \ldots, z_{d}\right]\right], f=z_{0}^{4}+z_{1}^{3}+z_{2}^{2}+\cdots+z_{d}^{2}$ and $R=A /(f)$, the $E_{6}$-singularity in dimension $d$. Then $|\mathrm{CM}(R)|_{z_{i}}=2$ for $i=0,1$ and $|\mathrm{CM}(R)|_{z_{i}}=1$ for $i=2, \ldots, d$. Moreover $|\mathrm{CM}(R)|_{t} \leq 2$ for any $t$ in $\underline{m}$.

The matrix factorizations for the other simple isolated singularities $\mathrm{D}_{n}, \mathrm{E}_{7}$ and $\mathrm{E}_{8}$ are listed in [13, 9.11-12, 9.14-15] and using the same methods as above we leave it to the reader to verify the following result.

Proposition 4.5 Let $A=k\left[\left[z_{0}, z_{1}, \ldots, z_{d}\right]\right], f$ in $A$ and $R=A /(f)$.

(a) For the $D_{n}$-singularity $f=z_{0}^{2} z_{1}+z_{1}^{n-1}+z_{2}^{2}+\cdots+z_{d}^{2}$ we have

$$
|\mathrm{CM}(R)|_{t}=\left\{\begin{array}{l}
2, t=z_{0} \\
1, t=z_{0} z_{1}, z_{2}, \ldots, z_{d} \\
{\left[\frac{n}{2}\right], t=z_{1}}
\end{array}\right.
$$

(b) For the $E_{7}$-singularity $f=z_{0}^{3}+z_{0} z_{1}^{3}+z_{2}^{2}+\cdots+z_{d}^{2}$ we have

$$
|\mathrm{CM}(R)|_{t}=\left\{\begin{array}{l}
2, t=z_{0} \\
1, t=z_{0} z_{1}, z_{2}, \ldots, z_{d} \\
3, t=z_{1}
\end{array}\right.
$$

(c) For the $E_{8}$-singularity $f=z_{0}^{5}+z_{1}^{3}+z_{2}^{2}+\cdots+z_{d}^{2}$ we have

$$
|\mathrm{CM}(R)|_{t}=\left\{\begin{array}{l}
3, t=z_{0} \\
1, t=z_{0} z_{1}, z_{2}, \ldots, z_{d} \\
2, t=z_{1}
\end{array}\right.
$$

\section{Acknowledgment}

The authors want to thank Professor Maurice Auslander for his interest and comments during the preparation of this paper. The second author also want to thank Professor Maurice Auslander and the Department of Mathematics at Brandeis University for their hospitality during his stay there 1990-91. 


\section{References}

[1] M. Auslander, Minimal Cohen-Macaulay approximations, in preparation.

[2] M. Auslander, R.-O. Buchweitz, The homological theory of maximal Cohen-Macaulay approximations, Soc. Math. de France, Mem. No. 38 (1989) 5-37.

[3] M. Auslander, S. Ding, Ø. Solberg, Liftings and weak liftings of modules, Preprint Math. No. 2, 1990, Departments of Mathematics, The University of Trondheim.

[4] C. W. Curtis, I. Reiner, Methods of representation theory, John Wiley \& Sons, New York, 1981.

[5] E. Dieterich, A. Wiedemann, The Auslander-Reiten quiver of a simple curve singularity, Trans. Amer. Math. Soc. 294 (1986) 455-475.

[6] D. Eisenbud, Homological algebra on a complete intersection with an application to group representations, Trans. Amer. Math. Soc. 260 (1980) 35-64.

[7] P. Hilton, D. Rees, Natural maps of extension functors and a theorem of R. G. Swan, Proc. Camb. Phil. Soc. Math. Phys. Sci. 51 (1961).

[8] H. Knörrer, Cohen-Macaulay modules on hypersurface singularities I, Invent. Math. 88 (1987) 153-164.

[9] T. Miyata, Note on direct summands of modules, J. Math. Kyoto Univ. 7 vol. 1 (1967) 65-69.

[10] K. W. Roggenkamp, Lattices over orders II, Lec. Notes in Math. (Springer 1970) 142.

[11] Ø. Solberg, Hypersurface singularities of finite Cohen-Macaulay type, London Math. Soc. (3) 58 (1989) 258-280.

[12] P. Symonds, The reduction of an RG-lattices modulo $p^{n}$, Can. J. of Math. vol. 42 No. 2 (1989) 342-364.

[13] Y. Yoshino, Cohen-Macaulay modules over Cohen-Macaulay rings, London Math. Soc. Lec. Notes Series 146. 